\title{
Spectral analysis of an abstract pair interaction model
}

\author{
Keisuke ASAHARA * Daiju FUNAKAWA ${ }^{\dagger}$
}

\begin{abstract}
We consider an abstract pair-interaction model in quantum field theory with a coupling constant $\lambda \in \mathbb{R}$ and analyze the Hamiltonian $H(\lambda)$ of the model. In the massive case, there exist constants $\lambda_{\mathrm{c}}<0$ and $\lambda_{\mathrm{c}, 0}<\lambda_{\mathrm{c}}$ such that, for each $\lambda \in$ $\left(\lambda_{c, 0}, \lambda_{\mathrm{c}}\right) \cup\left(\lambda_{\mathrm{c}}, \infty\right), H(\lambda)$ is diagonalized by a proper Bogoliubov transformation, so that the spectrum of $H(\lambda)$ is explicitly identified, where the spectrum of $H(\lambda)$ for $\lambda>\lambda_{\mathrm{c}}$ is different from that for $\lambda \in\left(\lambda_{\mathrm{c}, 0}, \lambda_{\mathrm{c}}\right)$. As for the case $\lambda<\lambda_{\mathrm{c}, 0}$, we show that $H(\lambda)$ is unbounded from above and below. In the massless case, $\lambda_{\mathrm{c}}$ coincides with $\lambda_{\mathrm{c}, 0}$.

Key words: quantum field, pair-interaction model, spectral analysis, Bogoliubov transformation.
\end{abstract}

2010 Mathematics Subject Classification: 47N50, 47B25, 81T10.

\section{Introduction}

In this paper, we consider an abstract pair-interaction model in quantum field theory. The Hamiltonian of the model is of the form

$$
H(\lambda):=\mathrm{d} \Gamma_{\mathrm{b}}(T)+\frac{\lambda}{2} \Phi_{\mathrm{s}}(g)^{2}
$$

acting in the boson Fock space $\mathscr{F}_{\mathrm{b}}(\mathscr{H})$ over a Hilbert space $\mathscr{H}$ (see Subsection 2.1), where $T$ is a self-adjoint operator on $\mathscr{H}, \mathrm{d} \Gamma_{\mathrm{b}}(T)$ is the second quantization operator of $T, \Phi_{\mathrm{s}}(g)$ is

${ }^{*}$ Graduate School of Science, Department of Mathematics, Hokkaido University

Kita 10, Nishi 8, Kita-Ku, Sapporo, Hokkaido, 060-0810, Japan

E-mail: asahara@math.sci.hokudai.ac.jp

${ }^{\dagger}$ Department of Electronics and Information Engineering, Faculty of Engineering, Hokkai-Gakuen University

4-1-40, Asahi-Cho, Toyohira-Ku, Sapporo, Hokkaido, 062-8605, Japan

E-mail: funakawa@hgu.jp 
the Segal field operator with test vector $g$ in $\mathscr{H}$ (see Subsection 2.1) and $\lambda \in \mathbb{R}$ is a coupling constant. A model of this type is called a $\phi^{2}$-model.

There have been many studies on massive or massless $\phi^{2}$-models in concrete forms or abstract forms (see, e.g., [4, 7, 8, 10, 11, 15]). In [10] and [15], the (essential) self-adjointness of the Hamiltonian of a $\phi^{2}$-model is proved in the case where $\lambda>0$ or $|\lambda|$ is sufficiently small. In [10], the existence of a ground state of a $\phi^{2}$-model also is shown in the case where the quantum field under consideration is massive and $\lambda>0$.

It is well known that Hamiltonians with linear and/or quadratic interactions in quantum fields may be analyzed by the method of Bogoliubov transformations (see, e.g., [1, 2, 3, 4, 6, 7, 9, 11]). A typical Bogoliubov transformation is constructed from bounded linear operators $U, V$ and a conjugation operator $J$ on $\mathscr{H}$ satisfying the following equations:

$$
\left\{\begin{aligned}
U^{*} U-V^{*} V & =I, \\
U_{J}^{*} V-V_{J}^{*} U & =0, \\
U U^{*}-V_{J} V_{J}^{*} & =I, \\
U V^{*}-V_{J} U_{J}^{*} & =0,
\end{aligned}\right.
$$

where $A_{J}:=J A J$ and $A^{*}$ is the adjoint of a densely defined linear operator $A$. It is well known that there is a unitary operator $\mathbb{U}$ on $\mathscr{F}_{\mathrm{b}}(\mathscr{H})$ which implements the Bogoliubov transformation in question if and only if $V$ is Hilbert-Schmidt [6, 12, 13, 14]. Moreover, it is shown that, under the condition that $V$ is Hilbert-Schmidt and suitable additional conditions, the Hamiltonian under consideration is unitarily equivalent via $\mathbb{U}$ to a second quantization operator up to a constant addition. For example, the Pauli-Fierz model with dipole approximation, which can be regarded as a kind of $\phi^{2}$-model, is analyzed by this method in 9 .

Recently, a general quadratic form Hamiltonian with a coupling constant $\lambda \in \mathbb{R}$ has been analyzed in [11] and it is shown that, in the case of a massive quantum field, under suitable conditions, the Hamiltonian is diagonalized by a Bogoliubov transformation. In [7], the sufficient condition formulated in [11] to obtain the result just mentioned has been extended. The spectrum of the standard pair-interaction model in physics, which is a concrete realization of the abstract pair-interaction model, is formally known [8] in the case where $\lambda>\lambda_{\mathrm{c}, 0}$ and $\lambda \neq \lambda_{\mathrm{c}}$ for some constants $\lambda_{\mathrm{c}}$ and $\lambda_{\mathrm{c}, 0}<\lambda_{\mathrm{c}}$. The paper [4] gives a rigorous proof for that in the framework of the boson Fock space theory over $\mathscr{H}=L^{2}\left(\mathbb{R}^{d}\right)$ for any $d \in \mathbb{N}$ and $\lambda>\lambda_{\mathrm{c}}$.

One of the motivations for the present work is to extend the theory developed in [4] with $\mathscr{H}=L^{2}\left(\mathbb{R}^{d}\right)$ to the theory with $\mathscr{H}$ being an abstract Hilbert space including the case where $\lambda<\lambda_{\text {c }}$. 
It is known [8] that spectral properties of a pair-interaction model may depend on the range of $\lambda$ with $\lambda_{\mathrm{c}}$ being a border point. Hence it is important to make this aspect clear mathematically. Therefore we analyze our model also for the region $\lambda<\lambda_{\text {c }}$. We show that, in the massive case with $\lambda \in\left(\lambda_{c, 0}, \lambda_{c}\right)$ also, the method of Bogoliubov transformations can be applied to prove that the Hamiltonian $H(\lambda)$ is unitarily equivalent to a second quantization operator up to a constant addition. Then we see that the spectrum of $H(\lambda)$ for $\lambda \in\left(\lambda_{c, 0}, \lambda_{c}\right)$ is different from that for $\lambda>\lambda_{\mathrm{c}}$. In the massless case, $\lambda_{\mathrm{c}, 0}$ coincides with $\lambda_{0}$.

The main results of the present paper include the following (1)-(3) (see Theorem 2.8 for more details): (1) Identification of the spectra of $H(\lambda)$ for $\lambda>\lambda_{c}$. (2) Identification of the spectra of $H(\lambda)$ for $\lambda_{\mathrm{c}, 0}<\lambda<\lambda_{\mathrm{c}}$ (the massive case; in the massless case, $\lambda_{\mathrm{c}, 0}=\lambda_{\mathrm{c}}$ ). In this case, bound states different from the ground state appear. (3) Unboundedness from above and below of $H(\lambda)$ for $\lambda<\lambda_{\mathrm{c}, 0}$.

The outline of this paper is as follows. In Section 2, we define our model and recall a fundamental fact in a general theory of Bogoliubov transformations. We prove the (essential) self-adjointness of $H(\lambda)$ (Theorem 2.3). Then we state the main theorem of this paper (Theorem 2.10). In Section 3, we construct operators $U$ and $V$ which are used to define the Bogoliubov transformation we need. In Section 4, we show that $U$ and $V$ satisfy (1.1) and $V$ is Hilbert-Schmidt. In Section 5. we prove Theorem 2.8 (1) and calculate the ground state energy of $H(\lambda)$ in the case $\lambda>\lambda_{c}$. In Section 6, we prove Theorem 2.8(2). In Section 7, we prove Theorem 2.8 (3). In Section 8, we consider a slightly generalized Hamiltonian of the form $H(\eta, \lambda):=H(\lambda)+\eta \Phi_{\mathrm{S}}(f)$ with $\eta \in \mathbb{R}$ and $f \in \mathscr{H}$. Applying the methods and results in the preceding sections, we can analyze $H(\eta, \lambda)$ to identify the spectra of it. In Appendix, we state some basic facts in the theory of boson Fock space.

\section{Preliminaries}

\subsection{The abstract Boson Fock Space}

Let $\mathscr{H}$ be a Hilbert space over the complex field $\mathbb{C}$ with the inner product $\langle\cdot, \cdot\rangle_{\mathscr{H}}$. The inner product is linear in the second variable and anti-linear in the first one. The symbol $\|\cdot\|_{\mathscr{H}}$ denotes the norm associated with it. We omit $\mathscr{H}$ in $\langle\cdot, \cdot\rangle_{\mathscr{H}}$ and $\|\cdot\|_{\mathscr{H}}$, respectively if

there is no danger of confusion. For each non-negative integer $n=0,1,2, \ldots, \otimes_{\mathrm{s}}^{n} \mathscr{H}$ denotes the $n$-fold symmetric tensor product Hilbert space of $\mathscr{H}$ with convention $\otimes_{\mathrm{s}}^{0} \mathscr{H}:=\mathbb{C}$. Then

$$
\mathscr{F}_{\mathrm{b}}(\mathscr{H}):=\oplus_{n=0}^{\infty} \otimes_{\mathrm{s}}^{n} \mathscr{H}
$$


is called the Boson-Fock space over $\mathscr{H}$. For a dense subspace $\mathscr{D}$ in $\mathscr{H}, \hat{\otimes}_{\mathrm{s}}^{n} \mathscr{D}$ denotes the algebraic $n$-fold symmetric tensor product of $\mathscr{D}$ with $\hat{\otimes}_{\mathrm{s}}^{0} \mathscr{H}:=\mathbb{C}$. Then

$$
\mathscr{F}_{\mathrm{b}, \text { fin }}(\mathscr{D}):=\hat{\oplus}_{n=0}^{\infty} \hat{\otimes}_{\mathrm{s}}^{n} \mathscr{D}
$$

is a dense subspace of $\mathscr{F}_{\mathrm{b}}(\mathscr{H})$, where $\hat{\oplus}_{n=0}^{\infty} \mathscr{D}_{n}$ denotes the algebraic direct sum of subspace $\mathscr{D}_{n} \subset \otimes_{\mathrm{s}}^{n} \mathscr{H}, n=0,1,2, \ldots$. The finite particle vector subspace

$\mathscr{F}_{\mathrm{b}, 0}(\mathscr{H}):=\left\{\psi=\left\{\psi^{(n)}\right\}_{n=0}^{\infty} \in \mathscr{F}_{\mathrm{b}}(\mathscr{H}) \mid \begin{array}{c}\psi^{(n)} \in \otimes_{\mathrm{s}}^{n} \mathscr{H}, n \geq 0, \text { there is an integer } \mathrm{n}_{0} \in \mathbb{N} \\ \text { such that } \psi^{(\mathrm{n})}=0, \text { for all } \mathrm{n} \geq \mathrm{n}_{0}\end{array}\right\}$

satisfies $\mathscr{F}_{\mathrm{b}, \text { fin }}(\mathscr{D}) \subset \mathscr{F}_{\mathrm{b}, 0}(\mathscr{H}) \subset \mathscr{F}_{\mathrm{b}}(\mathscr{H})$. It is dense in $\mathscr{F}_{\mathrm{b}}(\mathscr{H})$. For a linear operator $T$ on a Hilbert space, we denote its domain by $D(T)$.

For a densely defined closable operator $T$ on $\mathscr{H}$, let $T_{\mathrm{b}}^{(n)}$ be the densely defined closed operator on $\otimes_{\mathrm{s}}^{n} \mathscr{H}$ defined by

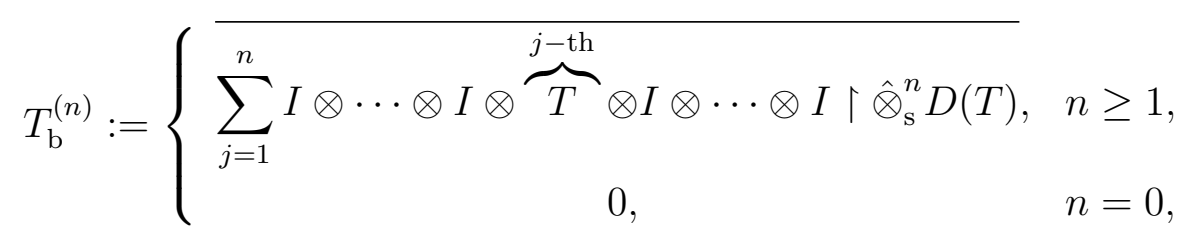

where $I$ denotes the identity operator on $\mathscr{H}, \bar{A}$ denotes the closure of a closable operator $A$ and $A \uparrow \mathcal{M}$ denotes the restriction of a linear operator $A$ on a subspace $\mathcal{M}$. The operator

$$
\mathrm{d} \Gamma_{\mathrm{b}}(T):=\oplus_{n=0}^{\infty} T_{\mathrm{b}}^{(n)}
$$

is called the second quantization operator of $T$. If $T$ is self-adjoint or non-negative, then so is $\mathrm{d} \Gamma_{\mathrm{b}}(T)$. For each $f \in \mathscr{H}$, there exists a unique densely defined closed operator $A(f)$ on $\mathscr{F}_{\mathrm{b}}(\mathscr{H})$ such that its adjoint $A(f)^{*}$ is given as follows:

$$
\begin{gathered}
D\left(A(f)^{*}\right):=\left\{\psi=\left\{\psi^{(n)}\right\}_{n=0}^{\infty} \in \mathscr{F}_{\mathrm{b}}(\mathscr{H}) \mid \sum_{n=0}^{\infty} n\left\|S_{n}\left(f \otimes \psi^{(n-1)}\right)\right\|^{2}<\infty\right\}, \\
\left(A(f)^{*} \psi\right)^{(n)}=\sqrt{n} S_{n}\left(f \otimes \psi^{(n-1)}\right), n \in \mathbb{N}, \quad\left(A(f)^{*} \psi\right)^{(0)}=0 \text { for } \psi \in D\left(A(f)^{*}\right),
\end{gathered}
$$

where $S_{n}$ is the symmetrization operator on the $n$-fold tensor product $\otimes^{n} \mathscr{H}$ of $\mathscr{H}$. The operator $A(f)$ (resp. $\left.A(f)^{*}\right)$ is called the annihilation (resp. creation) operator with test vector $f$. We have

$$
\mathscr{F}_{\mathrm{b}, 0}(\mathscr{H}) \subset D(A(f)) \cap D\left(A(f)^{*}\right)
$$

for all $f \in \mathscr{H}$ and $A(f)$ and $A(f)^{*}$ leave $\mathscr{F}_{\mathrm{b}, 0}(\mathscr{H})$ invariant. Moreover, they satisfy the following commutation relations:

$$
\left[A(f), A(g)^{*}\right]=\langle f, g\rangle, \quad[A(f), A(g)]=0, \quad\left[A(f)^{*}, A(g)^{*}\right]=0, \quad \text { for all } f, g \in \mathscr{H}
$$


on $\mathscr{F}_{\mathrm{b}, 0}(\mathscr{H})$, where $[A, B]:=A B-B A$ is the commutator of linear operators $A$ and $B$. The relation (2.1) is called the canonical commutation relations (CCR) over $\mathscr{H}$. The symmetric operator

$$
\Phi_{\mathrm{s}}(f):=\frac{1}{\sqrt{2}}\left(A(f)+A(f)^{*}\right), f \in \mathscr{H}
$$

is called the Segal-field operator with test vector $f$. We write its closure by the same symbol.

\subsection{Bogoliubov Transformation}

In this subsection, we define a Bogoliubov transformation and recall an important theorem about it. For a conjugation $J$ on $\mathscr{H}$ (i.e., $J$ is an anti-linear operator on $\mathscr{H}$ satisfying $\|J f\|=\|f\|$ for all $f \in \mathscr{H}$ and $J^{2}=I$ ) and a linear operator $A$ on $\mathscr{H}$, we define

$$
A_{J}:=J A J
$$

Definition 2.1. Let $U$ and $V$ be bounded linear operators on $\mathscr{H}$ and $J$ be a conjugation on $\mathscr{H}$. Then, for each $f \in \mathscr{H}$, we define a linear operator $B(f)$ on $\mathscr{F}_{\mathrm{b}}(\mathscr{H})$ by

$$
B(f):=A(U f)+A(J V f)^{*}
$$

Then the correspondence $\left(A(\cdot), A(\cdot)^{*}\right) \mapsto\left(B(\cdot), B(\cdot)^{*}\right)$ is called a Bogoliubov transformation.

By $\mathscr{F}_{\mathrm{b}, 0}(\mathscr{H}) \subset D(B(f))$, the adjoint $B(f)^{*}$ exists and the equation $B(f)^{*}=A(U f)^{*}+$ $A(J V f)$ holds on $\mathscr{F}_{\mathrm{b}, 0}(\mathscr{H})$ for each $f \in \mathscr{H}$. If the equations

$$
U^{*} U-V^{*} V=I, \quad U_{J}^{*} V-V_{J}^{*} U=0
$$

hold, then the Bogoliubov transformation preserves CCR, i.e., it holds that

$$
\left[B(f), B(g)^{*}\right]=\langle f, g\rangle,[B(f), B(g)]=0,\left[B(f)^{*}, B(g)^{*}\right]=0, \text { for all } f, g \in \mathscr{H}
$$

on $\mathscr{F}_{\mathrm{b}}(\mathscr{H})$. The following theorem is well-known [13, 14]:

Theorem 2.2. Let $\mathscr{H}$ be separable and $U$ and $V$ satisfy (1.1). Then there exists a unitary operator $\mathbb{U}$ on $\mathscr{F}_{\mathrm{b}}(\mathscr{H})$ such that

$$
\mathbb{U} \overline{B(f)} \mathbb{U}^{-1}=A(f), \quad f \in \mathscr{H}
$$

if and only if $V$ is Hilbert-Schmidt. 


\subsection{Hamiltonian}

For a self-adjoint operator $T$ on $\mathscr{H}$, constants $\lambda, \eta \in \mathbb{R}$ which are called coupling constants, and vectors $f, g \in \mathscr{H}$, we define an operator

$$
H(\lambda):=\mathrm{d} \Gamma_{\mathrm{b}}(T)+\frac{\lambda}{2} \Phi_{\mathrm{s}}(g)^{2}, \quad H(\eta, \lambda):=H(\lambda)+\eta \Phi_{\mathrm{s}}(f) .
$$

If $g \in D\left(T^{-1 / 2}\right)$, we define the constant

$$
\lambda_{\mathrm{c}, 0}:=-\left\|T^{-1 / 2} g\right\|^{-2}
$$

Theorem 2.3. Suppose that $T$ is an injective, non-negative, self-adjoint operator on $\mathscr{H}$. Let $f \in D\left(T^{-1 / 2}\right)$ and $g \in D\left(T^{-1 / 2}\right) \cap D(T)$. Then the following (1)-(3) hold:

(1) Let

$$
\lambda_{T}(g):=\left\|T^{-1 / 2} g\right\|^{-1}\left(\left\|T^{-1 / 2} g\right\|+\left\|T^{1 / 2} g\right\|\right)^{-1}
$$

and $|\lambda|<\lambda_{T}(g)$. Then $H(\eta, \lambda)$ is self-adjoint with $D(H(\eta, \lambda))=D\left(\mathrm{~d} \Gamma_{\mathrm{b}}(T)\right)$ and essentially self-adjoint on any core of $\mathrm{d} \Gamma_{\mathrm{b}}(T)$ for all $\eta \in \mathbb{R}$. Moreover $H(\eta, \lambda)$ is bounded from below.

(2) Let $|\lambda| \geq \lambda_{T}(g)$ and $f \in D\left(T^{1 / 2}\right)$. Then $H(\eta, \lambda)$ is essentially self-adjoint on any core of $\mathrm{d} \Gamma_{\mathrm{b}}(T)$ for all $\eta \in \mathbb{R}$. Moreover, if $\lambda \geq \lambda_{T}(g)$, then $H(\eta, \lambda)$ is self-adjoint.

(3) Let $f \in D\left(T^{1 / 2}\right)$. Then $\overline{H\left(\lambda_{\mathrm{c}, 0}\right)}$ is bounded from below. Moreover, if $\lambda>\lambda_{\mathrm{c}, 0}$, then $\overline{H(\eta, \lambda)}$ is bounded from below for all $\eta \in \mathbb{R}$ and $\left.D\left(\mathrm{~d} \Gamma_{\mathrm{b}}(T)^{1 / 2}\right)=D(\overline{H(\eta, \lambda)}+M)^{1 / 2}\right)$ for a constant $M \geq 0$ satisfying $\overline{H(\eta, \lambda)}+M \geq 0$.

Proof. (1) For any $\lambda \in \mathbb{R}$, by using (2.1), 9.1), (9.2) and [5, Theorem 5.18.], one can easily see that there are constants $a, b \geq 0$ such that for all $\psi \in D\left(\mathrm{~d} \Gamma_{\mathrm{b}}(T)\right)$,

$$
\left\|\frac{\lambda}{2} \Phi_{\mathrm{s}}(g)^{2} \psi\right\| \leq \frac{|\lambda|}{4}\left(a\left\|\mathrm{~d} \Gamma_{\mathrm{b}}(T) \psi\right\|+b\|\psi\|\right) .
$$

In particular, we can choose $a$ and $b$ which satisfy $a|\lambda| / 4<1$ if $|\lambda|<\lambda_{T}(g)$. We remark that, to obtain the factor $\lambda_{T}(g)$, we need to deform terms $\left\|A(g)^{* 2} \psi\right\|^{2},\left\|A(g)^{*} A(g) \psi\right\|^{2}$ and $\left\|A(g)^{2} \psi\right\|^{2}$ coming from $\left\|\Phi_{\mathrm{s}}(g) \psi\right\|^{2}\left(\psi \in \mathscr{F}_{\mathrm{b}, 0}(\mathscr{H})\right)$ to $\left\|A(g) A(g)^{*} \psi\right\|^{2}+$ a marginal term respectively. Thus, for $|\lambda|<\lambda_{T}(g)$, by the Kato-Rellich theorem, $H(\lambda)$ is selfadjoint. It is well known that $\Phi_{\mathrm{s}}(f)$ is infinitesimally small with respect to $\mathrm{d} \Gamma_{\mathrm{b}}(T)$. Hence, by the Kato-Rellich theorem, for $|\lambda|<\lambda_{T}(g), H(\eta, \lambda)$ is self-adjoint. 
(2) Firstly, we show that, for any $f \in D\left(T^{1 / 2}\right)$ and $\eta, \lambda \in \mathbb{R}, H(\eta, \lambda)$ is essentially selfadjoint on any core of $\mathrm{d} \Gamma_{\mathrm{b}}(T)$. By (9.1), (9.2) and [5, Theorem 5.18.], we can see that there exists $a>0$ such that $\|H(\eta, \lambda) \psi\| \leq a\left\|\left(\mathrm{~d} \Gamma_{\mathrm{b}}(T)+I\right) \psi\right\|$ for all $\psi \in D\left(\mathrm{~d} \Gamma_{\mathrm{b}}(T)\right)$. For the first let $f \in D(T)$. Then by (2.1) and (9.3), for any $\psi \in \mathscr{F}_{\mathrm{b}, \text { fin }}(D(T))$, we have

$$
\begin{aligned}
& \left\langle H(\eta, \lambda) \psi,\left(\mathrm{d} \Gamma_{\mathrm{b}}(T)+I\right) \psi\right\rangle-\left\langle\left(\mathrm{d} \Gamma_{\mathrm{b}}(T)+I\right) \psi, H(\eta, \lambda) \psi\right\rangle \\
= & \frac{\lambda}{\sqrt{2}}\left(\left\langle\Phi_{\mathrm{s}}(g) \psi, A(T g) \psi\right\rangle-\left\langle A(T g) \psi, \Phi_{\mathrm{s}}(g) \psi\right\rangle\right)+\frac{\eta}{\sqrt{2}}(\langle\psi, A(T f) \psi\rangle-\langle A(T f) \psi, \psi\rangle) .
\end{aligned}
$$

Thus, by (9.1) and (9.2), we have

$$
\left|\left\langle H(\eta, \lambda) \psi,\left(\mathrm{d} \Gamma_{\mathrm{b}}(T)+I\right) \psi\right\rangle-\left\langle\left(\mathrm{d} \Gamma_{\mathrm{b}}(T)+I\right) \psi, H(\eta, \lambda) \psi\right\rangle\right| \leq C\left\|\left(\mathrm{~d} \Gamma_{\mathrm{b}}(T)+I\right)^{1 / 2} \psi\right\|^{2},
$$

where $C:=\left\{|\lambda||| T^{1 / 2} g\left\|\left(\|g\|+2\left\|T^{-1 / 2} g\right\|\right)+\sqrt{2}|\eta|\right\| T^{1 / 2} f \|\right\}$. By a limiting argument, using the fact that $\mathscr{F}_{\mathrm{b}, \text { fin }}(D(T))$ is a core of $\mathrm{d} \Gamma_{\mathrm{b}}(T)$ and $\mathrm{d} \Gamma_{\mathrm{b}}(T)$-boundedness of $\Phi_{\mathrm{s}}(g)^{2}$, we can show that for $f \in D\left(T^{1 / 2}\right)$ and $\psi \in D\left(\mathrm{~d} \Gamma_{\mathrm{b}}(T)\right)$, 2.3p holds. Thus, by the Nelson commutator theorem, for all $\eta, \lambda \in \mathbb{R}, H(\eta, \lambda)$ is essentially self-adjoint and $\overline{H(\eta, \lambda)}$ is essentially self-adjoint on any core of $\mathrm{d} \Gamma_{\mathrm{b}}(T)$. The equation $\overline{H(\eta, \lambda) \uparrow \mathscr{D}}=\overline{\overline{H(\eta, \lambda)}\lceil\mathscr{D}}$ holds for any core $\mathscr{D}$ of $\mathrm{d} \Gamma_{\mathrm{b}}(T)$. Hence $H(\eta, \lambda)$ is essentially self-adjoint on any core of $\mathrm{d} \Gamma_{\mathrm{b}}(T)$ for all $\eta, \lambda \in \mathbb{R}$. Next we show that, if $\lambda>-\left\|T^{-1 / 2} g\right\|^{-1}\left(\left\|T^{-1 / 2} g\right\|+\left\|T^{1 / 2} g\right\|\right)^{-1}$, then $H(\eta, \lambda)$ is self-adjoint. We can show that, for $\lambda>0$ and any $0<\varepsilon<1$, there is a constant $c_{\varepsilon}>0$ such that

$$
(1-\varepsilon)\left\|\mathrm{d} \Gamma_{\mathrm{b}}(T) \psi\right\|^{2}+\left\|\frac{\lambda}{2} \Phi_{\mathrm{s}}(g)^{2} \psi\right\|^{2} \leq\|H(\eta, \lambda) \psi\|^{2}+c_{\varepsilon}\|\psi\|^{2}, \quad \psi \in D\left(\mathrm{~d} \Gamma_{\mathrm{b}}(T)\right) .
$$

Hence $H(\eta, \lambda)$ is closed. In particular, it is self-adjoint.

(3) It is well known that, for any $\varepsilon>0, \varepsilon \mathrm{d} \Gamma_{\mathrm{b}}(T)+\eta \Phi_{\mathrm{s}}(f)$ is bounded from below. For any $\varepsilon>0$ and $\psi \in D\left(\mathrm{~d} \Gamma_{\mathrm{b}}(T)^{1 / 2}\right)$,

$$
|\langle\psi, A(f) \psi\rangle| \leq\left\|T^{-1 / 2} f\right\|\left(\varepsilon\left\|\mathrm{d} \Gamma_{\mathrm{b}}(T)^{1 / 2} \psi\right\|^{2}+\frac{1}{4 \varepsilon}\|\psi\|^{2}\right) .
$$

Hence if the assertion follows for $\eta=0$, then so is for all $\eta$. Thus we show that the assertion follows for $\eta=0$. If $\lambda>0$, then clearly $H(\lambda) \geq 0$. Let $\lambda<0$. For any $\psi \in D\left(\mathrm{~d} \Gamma_{\mathrm{b}}(T)^{1 / 2}\right)$,

$$
\left\|\Phi_{\mathrm{s}}(g) \psi\right\|^{2} \leq 2\left\|T^{-1 / 2} g\right\|^{2}\left\|\mathrm{~d} \Gamma_{\mathrm{b}}(T)^{1 / 2} \psi\right\|^{2}+\|g\|^{2}\|\psi\|^{2} .
$$


Thus for any $\psi \in D\left(\mathrm{~d} \Gamma_{\mathrm{b}}(T)\right)$,

$$
\begin{aligned}
\langle\psi, H(\lambda) \psi\rangle & =\left\|\mathrm{d} \Gamma_{\mathrm{b}}(T)^{1 / 2} \psi\right\|^{2}+\frac{\lambda}{2}\left\|\Phi_{\mathrm{s}}(g) \psi\right\|^{2} \\
& \geq\left(1+\lambda\left\|T^{-1 / 2} g\right\|^{2}\right)\left\|\mathrm{d} \Gamma_{\mathrm{b}}(T)^{1 / 2} \psi\right\|^{2}+\frac{\lambda}{2}\|g\|^{2}\|\psi\|^{2} .
\end{aligned}
$$

Hence $H(\lambda)$ is bounded from below if $\lambda \geq \lambda_{c, 0}$.

Let $\lambda \geq \lambda_{c, 0}$ and $M \geq 0$ be a constant satisfying $H(\lambda)+M \geq 0$. Then for any $\psi \in D\left(\mathrm{~d} \Gamma_{\mathrm{b}}(T)\right)=D(H(\lambda))$,

$$
\left\|(\overline{H(\lambda)}+M)^{1 / 2} \psi\right\|^{2} \leq\left(1+|\lambda|\left\|T^{-1 / 2} g\right\|^{2}\right)\left\|\mathrm{d} \Gamma_{\mathrm{b}}(T)^{1 / 2} \psi\right\|^{2}+\left(\frac{|\lambda|}{2}\|g\|^{2}+M\right)\|\psi\|^{2} .
$$

By the fact that $D\left(\mathrm{~d} \Gamma_{\mathrm{b}}(T)\right)$ is a core of $\mathrm{d} \Gamma_{\mathrm{b}}(T)^{1 / 2}$, we have $D\left(\mathrm{~d} \Gamma_{\mathrm{b}}(T)^{1 / 2}\right) \subset D(\overline{H(\lambda)}+$ $M)^{1 / 2}$ ) and (2.5) holds on $D\left(\mathrm{~d} \Gamma_{\mathrm{b}}(T)^{1 / 2}\right)$.

In the case of $\lambda>0$, it is easy to see that $\left\|H(\lambda)^{1 / 2} \psi\right\| \geq\left\|\mathrm{d} \Gamma_{\mathrm{b}}(T)^{1 / 2} \psi\right\|$ holds for any $\psi \in D\left(\mathrm{~d} \Gamma_{\mathrm{b}}(T)\right)$. In the case of $0>\lambda>\lambda_{\mathrm{c}, 0}$,

$$
\left\|\mathrm{d} \Gamma_{\mathrm{b}}(T)^{1 / 2} \psi\right\|^{2} \leq \frac{1}{1+\lambda\left\|T^{-1 / 2} g\right\|^{2}}\left\{\left\|(\overline{H(\lambda)}+M)^{1 / 2} \psi\right\|^{2}-\left(\frac{\lambda}{2}\|g\|^{2}+M\right)\|\psi\|^{2}\right\} .
$$

holds for any $\psi \in D\left(\mathrm{~d} \Gamma_{\mathrm{b}}(T)\right)$ by (2.4). Hence for $\lambda>\lambda_{\mathrm{c}, 0}$ there is a constant $a, b \geq 0$ such that

$$
\left\|\mathrm{d} \Gamma_{\mathrm{b}}(T)^{1 / 2} \psi\right\| \leq a\left\|(\overline{H(\lambda)}+M)^{1 / 2} \psi\right\|+b\|\psi\| .
$$

By operational calculus, $D\left(\mathrm{~d} \Gamma_{\mathrm{b}}(T)\right)$ is a core of $(\overline{H(\lambda)}+M)^{1 / 2}$. Thus we have $D((\overline{H(\lambda)}+$ $\left.M)^{1 / 2}\right) \subset D\left(\mathrm{~d} \Gamma_{\mathrm{b}}(T)^{1 / 2}\right)$ and (2.6) holds on $D\left((\overline{H(\lambda)}+M)^{1 / 2}\right)$.

Remark 2.4. By [3, Lemma13-15], if $\mathscr{H}$ is separable, then Theorem 2.3 takes the following forms:

Let $\mathscr{H}$ be separable, $T$ be a non-negative, injective self-adjoint operator, $f \in D\left(T^{-1 / 2}\right)$ and $g \in D\left(T^{-1 / 2}\right) \cap D\left(T^{1 / 2}\right)$. Then the following (1)-(3) holds:

(1) Let $\lambda>\lambda_{c, 0}$. Then $H(\eta, \lambda)$ is self-adjoint with $D(H(\eta, \lambda))=D\left(\mathrm{~d} \Gamma_{\mathrm{b}}(T)\right)$ and essentially self-adjoint on any core of $\mathrm{d} \Gamma_{\mathrm{b}}(T)$ for all $\eta \in \mathbb{R}$. Moreover $H(\eta, \lambda)$ is bounded from below.

(2) Let $\lambda \leq \lambda_{c, 0}$ and $f \in D\left(T^{1 / 2}\right)$. Then $H(\eta, \lambda)$ is essentially self-adjoint on any core of $\mathrm{d} \Gamma_{\mathrm{b}}(T)$ for all $\eta \in \mathbb{R}$. In particular, if $\eta=0$ and $\lambda=\lambda_{\mathrm{c}, 0}$, then $H\left(\lambda_{\mathrm{c}, 0}\right)=H\left(0, \lambda_{\mathrm{c}, 0}\right)$ is bounded from below. 
(3) Let $\lambda>\lambda_{\mathrm{c}, 0}$. Then $\left.D\left(\mathrm{~d} \Gamma_{\mathrm{b}}(T)^{1 / 2}\right)=D(H(\eta, \lambda)+M)^{1 / 2}\right)$ for a constant $M \geq 0$ satisfying $H(\eta, \lambda)+M \geq 0$.

Definition 2.5. Let $T$ be a self-adjoint operator on $\mathscr{H}$ and $\left\{E(B) \mid B \in \mathbf{B}^{1}\right\}$ be the spectral measure associated with $T$ on the Borel field $\mathbf{B}^{1}$ on $\mathbb{R}$. The operator $T$ is called purely absolutely continuous if, for each $f \in \mathscr{H}$, the measure $\|E(\cdot) f\|^{2}$ on $\mathbf{B}^{1}$ is absolutely continuous with respect to the one-dimensional Lebesgue measure.

Definition 2.6. For a purely absolutely continuous self-adjoint operator $T$ and vectors $f, g \in$ $\mathscr{H}, \psi_{g, f}$ denotes the Radon-Nikodym derivative of the finite complex Borel measure $\langle g, E(\cdot) f\rangle$ on $\mathbf{B}^{1}$. In particular, we set $\psi_{g}:=\psi_{g, g}$.

\subsection{Assumptions}

To prove our main theorem stated later (Theorem 2.10), we need some assumptions. For a closed operator $A, \sigma(A)$ denotes the spectrum of $A$. If $A$ is self-adjont, then $\sigma_{\mathrm{ac}}(A)$ (resp. $\left.\sigma_{\mathrm{p}}(A), \sigma_{\mathrm{sc}}(A)\right)$ denotes the absolutely continuous (resp. point, singular continuous) spectrum of $A$. For a self-adjoint operator $A$ bounded from below,

$$
E_{0}(A):=\inf \sigma(A)
$$

is called the lowest energy of $A$. In particular, it is called the ground state energy of $A$ if $E_{0}(A) \in \sigma_{\mathrm{p}}(A)$. In this case, any for responding eigenvector is called a ground state of $A$. The ground state is said to be unique if $\operatorname{dim} \operatorname{Ker}\left(A-E_{0}(A)\right)=1$. For linear operators $A$ and $B$, the symbol $A \subset B$ means that $D(A) \subset D(B)$ and $A f=B f$ for all $f \in D(A)$, i.e., $B$ is an extension of $A$.

Assumption 2.7. (1) The operator $T$ is a non-negative, purely absolutely continuous selfadjoint operator,

(2) The fixed vector $g \in \mathscr{H}$ satisfies $g \in D\left(\hat{T}^{-1 / 2}\right) \cap D\left(T^{1 / 2}\right)$ and $J g=g$, where $\hat{T}:=T-E_{0}$, $E_{0}:=E_{0}(T)$ and $J$ is a conjugation on $\mathscr{H}$ satisfying $J D(T) \subset D(T)$ and $J T \psi=T J \psi$ for any $\psi \in D(T)$ ( i.e., $J T \subset T J)$,

(3) $\sup _{E_{0}<x} x^{ \pm 1} \psi_{g}(x)<\infty$ and $\psi_{g}(x)>0$ for all $x \in\left(E_{0}, \infty\right)$,

(4) $\psi_{g} \in C^{1}\left(\left(E_{0}, \infty\right)\right)$ and $\lim _{x \downarrow E_{0}} x^{-1} \psi_{g}^{\prime}(x)=0=\lim _{x \rightarrow \infty} x^{-1} \psi_{g}^{\prime}(x)$.

Remark 2.8. The operator $T$ is injective since it is a purely absolutely continuous selfadjoint operator. Since $T$ has no eigenvector, the inverse of $\hat{T}$ exists. Assumption 2.7 (2) 
implies that $T_{J}=T$. In general, for a self-adjoint operator $A$ and a conjugation $J$, we can choose a vector $f \in D(A)$ satisfying $J f=f$ if $A_{J}=A$. Thus the vector $g$ in Assumption 2.7 (2) exists. By Assumption 2.7 (3), one can easily show that $\sup _{x \in \sigma(T)} \psi_{g}(x)<\infty$ and, for each $f \in \mathscr{H}$, the functions $\psi_{g, f}, \psi_{T^{ \pm 1 / 2} g, f}$ are in $L^{2}(\mathbb{R})$ and the maps $: f \mapsto \psi_{g, f}, \psi_{T^{ \pm 1 / 2}{ }^{\prime}, f}$ are bounded. Actually, for any $h \in \mathscr{H}$ and $B \in \mathbf{B}^{1}$, the following inequality holds

$$
|\langle E(B) h, f\rangle|^{2} \leq\|E(B) h\|^{2}\|E(B) f\|^{2}
$$

by Schwarz's inequality. Thus we obtain $\left|\psi_{h, f}(\mu)\right|^{2} \leq \psi_{h}(\mu) \psi_{f}(\mu)$ for almost all $\mu \in \mathbb{R}$ with respect to the Lebesgue measure. Hence, by Assumption 2.7 (3), we have the boundedness of the mappings. Moreover, we see that for any $F \in L^{2}(\mathbb{R}), g \in D(F(T)$ ), where $F(T)$ denotes the operator defined by $F(T):=\int_{\mathbb{R}} F(\mu) d E(\mu)$. In particular, $g$ is in $D\left(\psi_{g, f}(T)\right)$ for any $f \in \mathscr{H}$.

Lemma 2.9. Let $T$ be a self-adjoint operator such that JT $\subset$ TJ. Then

(1) $E(B)_{J}=E(B)$, for all $B \in \mathbf{B}^{1}$.

(2) Let $F$ be a Borel measurable function on $\mathbb{R}$. Then $F(T)_{J}=F^{*}(T)$, where $F^{*}$ is complex conjugation of $F$.

Proof. These are proved by using the spectral theorem.

\subsection{The Main Theorem}

In this subsection, we state the main theorem of the present paper. Let $\lambda_{\mathrm{c}}$ be a constant defined by

$$
\lambda_{\mathrm{c}}:=-\left(\int_{\left[E_{0}, \infty\right)} \frac{\mu}{\mu^{2}-E_{0}^{2}} d\|E(\mu) g\|^{2}\right)^{-1}<0 .
$$

Then it is easy to see that $\lambda_{\mathrm{c}, 0} \leq \lambda_{\mathrm{c}}$, and $\lambda_{\mathrm{c}, 0}=\lambda_{\mathrm{c}}$ if and only if $E_{0}=0$.

Theorem 2.10. Let $\mathscr{H}$ be separable. Then the following (1)-(3) hold:

(1) Let $T$ and $g$ satisfy Assumption 2.7. If $\lambda>\lambda_{\mathrm{c}}$, then there are a unitary operator $\mathbb{U}$ on $\mathscr{F}_{\mathrm{b}}(\mathscr{H})$ and a constant $E_{\mathrm{g}} \in \mathbb{R}$ such that

$$
\mathbb{U} H(\lambda) \mathbb{U}^{-1}=\mathrm{d} \Gamma_{\mathrm{b}}(T)+E_{\mathrm{g}}
$$

In particular, $\mathbb{U}^{-1} \Omega_{0}$ is the unique ground state of $H(\lambda)$ up to constant multiples, and

$$
\begin{aligned}
\sigma(H(\lambda)) & =\left\{E_{\mathrm{g}}\right\} \cup\left[E_{0}+E_{\mathrm{g}}, \infty\right), \\
\sigma_{\mathrm{ac}}(H(\lambda)) & =\left[E_{0}+E_{\mathrm{g}}, \infty\right), \sigma_{\mathrm{p}}(H(\lambda))=\left\{E_{\mathrm{g}}\right\}, \sigma_{\mathrm{sc}}(H(\lambda))=\emptyset .
\end{aligned}
$$


(2) Let $T$ and $g$ satisfy Assumption 2.7 and $E_{0}>0$. If $\lambda_{c, 0}<\lambda<\lambda_{c}$, then there exist a unitary operator $\mathbb{V}$ on $\mathscr{F}_{\mathrm{b}}(\mathscr{H})$, an injective non-negative self-adjoint operator $\xi$ on $\mathscr{H}$ and a constant $E_{\mathrm{b}} \geq 0$ such that $\xi$ has a ground state and

$$
\mathbb{V} H(\lambda) \mathbb{V}^{-1}=\mathrm{d} \Gamma_{\mathrm{b}}(\xi)+E_{\mathrm{g}}-E_{\mathrm{b}} .
$$

In particular, $\mathbb{V}^{-1} \Omega_{0}$ is the unique ground state of $H(\lambda)$ up to constant multiples, and

$$
\begin{aligned}
& \sigma(H(\lambda))=\cup_{n=0}^{\infty}\left\{n \beta+E_{\mathrm{g}}-E_{\mathrm{b}}\right\} \cup\left[E_{0}+E_{\mathrm{g}}-E_{\mathrm{b}}, \infty\right), \\
& \sigma_{\mathrm{ac}}(H(\lambda))=\left[E_{0}+E_{\mathrm{g}}-E_{\mathrm{b}}, \infty\right), \\
& \sigma_{\mathrm{p}}(H(\lambda))=\cup_{n=0}^{\infty}\left\{n \beta+E_{\mathrm{g}}-E_{\mathrm{b}}\right\}, \sigma_{\mathrm{sc}}(H(\lambda))=\emptyset,
\end{aligned}
$$

where $\beta>0$ is the discrete ground state energy of $\xi$.

(3) Let $T$ be a non-negative, injective self-adjoint operator. If $g \in D\left(T^{-1 / 2}\right)$ and $\lambda<\lambda_{c, 0}$, then $H(\lambda)$ is unbounded from above and below.

Example 2.11. A concrete realization of the abstract model is given as follows (see [8, Chapter 12]):

$$
\mathscr{H} \leftrightarrow L^{2}\left(\mathbb{R}^{d}\right), \quad T \leftrightarrow \omega, \quad g \leftrightarrow \frac{\hat{\rho}}{\sqrt{\omega}}
$$

where $\omega$ is a multiplication operator associated with the function $\omega(k):=\sqrt{|k|^{2}+m^{2}}, k \in \mathbb{R}^{d}$ for a fixed $m \geq 0$ and $\hat{\rho}$ is the Fourier transform of a function $\rho \in L^{2}\left(\mathbb{R}^{d}\right)$ satisfying $\hat{\rho} / \sqrt{\omega} \in$ $L^{2}\left(\mathbb{R}^{d}\right)$. Assume that $\hat{\rho}$ is rotation invariant, i.e., there exists a function $v$ on $[0, \infty)$ such that $\hat{\rho}(k)=v(|k|)$ for all $k \in \mathbb{R}^{d}$. Then we have $\psi_{g}(s)=\left|S^{d-1}\right| \omega_{1}^{-1}(s)^{d-2}\left|v\left(\omega_{1}^{-1}(s)\right)\right|^{2}$ for $s \geq m$, where $\left|S^{d-1}\right|$ is the surface area of the $(d-1)$-dimensional unite sphere with convention $\left|S^{0}\right|=2 \pi$ and $\omega_{1}(r)=\sqrt{r^{2}+m^{2}}, r \geq 0$. Hence, with $J$ being the complex conjugation, the following conditions (2)'-(4)' imply that the present model satisfies Assumption 2.7 .

$(2)^{\prime} \hat{\rho}(k)^{*}=\hat{\rho}(k)$ and

$$
\hat{\rho} \in L^{2}\left(\mathbb{R}^{d}\right), \int_{\mathbb{R}^{d}} \frac{|\hat{\rho}(k)|^{2}}{|k|^{2}} d k<\infty .
$$

(3)' $\hat{\rho}$ is rotation invariant. $\sup _{k \in \mathbb{R}^{d}} \omega(k)^{ \pm 1 / 2}|k|^{(d-2) / 2}|\hat{\rho}(k)|<\infty$. $\hat{\rho}(k)>0$, for all $k \neq 0$.

(4)' $v \in C^{1}([0, \infty))$ and

$$
\begin{aligned}
& \lim _{|k| \rightarrow 0}|k|^{d-4} \hat{\rho}(k)\left\{(d-2) \hat{\rho}(k)+2 v^{\prime}(|k|)\right\}=0 . \\
& \lim _{|k| \rightarrow \infty}|k|^{d-4} \hat{\rho}(k)\left\{(d-2) \hat{\rho}(k)+2 v^{\prime}(|k|)\right\}=0 .
\end{aligned}
$$


For example, one can easily check that the function

$$
\hat{\rho}(k):=\exp \left(-\frac{1}{|k|^{2}}-|k|^{2}\right), k \in \mathbb{R}^{d} \backslash\{0\}, \hat{\rho}(0):=0
$$

satisfies the above conditions (2)'-(4)'.

\section{Definitions and properties of some functions and op- erators}

In this section we introduce some functions and operators. We assume that $\mathscr{H}$ is separable and Assumption 2.7 from this section to Section 6.

\subsection{Functions $D$ and $D_{ \pm}$}

Lemma 3.1. Let $D: \mathbb{C} \backslash(0, \infty) \rightarrow \mathbb{C}$ be the function

$$
D(z):=1+\lambda \int_{\left[E_{0}, \infty\right)} \frac{\mu}{\mu^{2}-E_{0}^{2}-z} d\|E(\mu) g\|^{2}, \quad z \in \mathbb{C} \backslash(0, \infty) .
$$

Then $D$ is well-defined and analytic in $\mathbb{C} \backslash[0, \infty)$. Moreover, the following hold:

(1) For all $\lambda>\lambda_{c}, D(z)$ has no zeros in $\mathbb{C} \backslash[0, \infty)$.

(2) For all $\lambda<\lambda_{c}, D(z)$ has a unique simple zero in the negative real axis $(-\infty, 0)$.

Proof. If $\operatorname{Im} z \neq 0$ (resp. $\operatorname{Re} z<0$ ), then for any $n \in \mathbb{N}$,

$$
\int_{\left[E_{0}, \infty\right)}\left|\frac{\mu}{\left(\mu^{2}-E_{0}^{2}-z\right)^{n}}\right| d\|E(\mu) g\|^{2} \leq c^{-n}\left\|T^{1 / 2} g\right\|^{2}<\infty,
$$

where $c$ is $|\operatorname{Re} z|(\operatorname{resp} .|\operatorname{Im} z|)$. If $z=0$, then

$$
\int_{\left[E_{0}, \infty\right)} \frac{\mu}{\mu^{2}-E_{0}^{2}} d\|E(\mu) g\|^{2} \leq\left\|\hat{T}^{-1 / 2} g\right\|^{2}<\infty .
$$

Thus, by using the Lebesgue dominated convergence theorem, $D$ is well-defined and analytic in $\mathbb{C} \backslash[0, \infty)$.

(1) If $\lambda=0$, then $D(z)=1$ for all $z \in \mathbb{C} \backslash(0, \infty)$, so it has no zeros. Let $\lambda \neq 0$ and $z=x+i y \in \mathbb{C} \backslash(0, \infty)$. Then we see that

$$
\operatorname{Im} D(z)=y \lambda \int_{\left[E_{0}, \infty\right)} \frac{\mu}{\left(\mu^{2}-E_{0}^{2}-x\right)^{2}+y^{2}} d\|E(\mu) g\|^{2} .
$$


Thus $\operatorname{Im} D(z)=0$ is equivalent to $y=0$. Therefore $D(z)=0$ if and only if $D(x)=0$. Let $y=0$. In the case $\lambda>0$, one has $D(x)>0$ for all $x \in(-\infty, 0]$. Thus $D$ has no zeros. Next, we consider the case $\lambda<0$. We have for $x<0$,

$$
D^{\prime}(x)=\lambda \int_{\left[E_{0}, \infty\right)} \frac{\mu}{\left(\mu^{2}-E_{0}^{2}-x\right)^{2}} d\|E(\mu) g\|^{2}<0 .
$$

Thus $D$ is monotone decreasing in $(-\infty, 0)$. If $\lambda>\lambda_{c}$, then $D(0)>0$. Hence $D$ has no zeros.

(2) Let $\lambda<\lambda_{c}$. We can see that

$$
D(0)=1+\lambda \int_{\left[E_{0}, \infty\right)} \frac{\mu}{\mu^{2}-E_{0}^{2}} d\|E(\mu) g\|^{2}=1-\frac{\lambda}{\lambda_{c}}<0
$$

By the Lebesgue dominated convergence theorem $D(x) \rightarrow 1$ as $x \rightarrow-\infty$. Since $D$ is monotone decreasing in $(-\infty, 0), D$ has a unique simple zero in $(-\infty, 0)$.

Let

$$
\phi_{g}(x):=\psi_{g}(\sqrt{x}) \chi_{\left[E_{0}^{2}, \infty\right)}(x), x \in \mathbb{R},
$$

where $\chi_{B}$ is the characteristic function of $B \in \mathbf{B}^{1}$.

Lemma 3.2. The following hold:

(1) The function $\phi_{g}$ satisfies $\phi_{g} \in C^{1}(\mathbb{R}) \cap L^{1}(\mathbb{R}) \cap L^{2}(\mathbb{R})$ and $\sup _{x \in \mathbb{R}}\left|\phi_{g}^{\prime}(x)\right|<\infty$.

(2) Let

$$
A_{\varepsilon}^{(1)}(x):=\frac{x}{\pi\left(x^{2}+\varepsilon^{2}\right)}, A_{\varepsilon}^{(2)}(x):=\frac{\varepsilon}{\pi\left(x^{2}+\varepsilon^{2}\right)}, x \in \mathbb{R}, \varepsilon>0
$$

be the conjugate poisson kernel and the poisson kernel respectively and $f * h$ denote the convolution of functions $f$ and $h$. Let

$$
\left(H_{\varepsilon} f\right)(s):=\frac{1}{\pi} \int_{|x-s| \geq \varepsilon} \frac{f(x)}{x-s} d x,(H f)(s):=\lim _{\varepsilon \downarrow 0}\left(H_{\varepsilon} f\right)(s), s \in \mathbb{R}, \varepsilon>0,
$$

where $H f$ is called the Hilbert transform of $f$. Then for all $x \in \mathbb{R}$,

$$
\lim _{\varepsilon \downarrow 0}\left(A_{\varepsilon}^{(1)} * \phi_{g}\right)(x)=\left(H \phi_{g}\right)(x), \quad \lim _{\varepsilon \downarrow 0}\left(A_{\varepsilon}^{(2)} * \phi_{g}\right)(x)=\phi_{g}(x),
$$

hold uniformly in $x$. 
Proof. By Assumption 2.7 (2), (3) and (4), the assertion (1) holds. Next we consider the assertion (2). By (1), in particular, $\phi_{g}$ is bounded and uniformly continuous. Thus it is easy to see that $A_{\varepsilon}^{(2)} * \phi_{g}$ converges uniformly to $\phi_{g}$. Moreover, by (1), Holder's inequality, the mean value theorem and a similar estimate to the proof of [16, Theorem 92.], we can show that $\left(A_{\varepsilon}^{(1)} * \phi_{g}\right)(x)-\left(H_{\varepsilon} \phi_{g}\right)(x)$ tends to 0 uniformly in $x$ as $\varepsilon \downarrow 0$. Hence the assertion (2) holds.

Detailed studies of the Hilbert transform are given in [16].

Lemma 3.3. For all $s \geq 0, D_{ \pm}(s):=\lim _{\varepsilon \downarrow 0} D(s \pm i \varepsilon)$ are uniformly convergent and continuous in $s \geq 0$ with

$$
D_{ \pm}(s)=1+\frac{\lambda \pi}{2}\left(H \phi_{g}\right)\left(E_{0}^{2}+s\right) \pm i \frac{\lambda \pi}{2} \psi_{g}\left(\sqrt{E_{0}^{2}+s}\right), \quad s \geq 0
$$

Proof. For any $s \geq 0$ and $\varepsilon>0$, we have by change of variable

$$
D(s \pm i \varepsilon)=\frac{\lambda \pi}{2}\left(A_{\varepsilon}^{(1)} * \phi_{g}\right)\left(E_{0}^{2}+s\right) \pm i \frac{\lambda \pi}{2}\left(A_{\varepsilon}^{(2)} * \phi_{g}\right)\left(E_{0}^{2}+s\right) .
$$

Thus, by Lemma 3.2, $D_{ \pm}$converge uniformly in $s \geq 0$ and 3.1 holds. The continuity of $D_{ \pm}$ is due to the uniform convergence.

Remark 3.4. For all $\mu \in\left[E_{0}, \infty\right)$, we have

$$
i \pi \lambda \psi_{g}(\mu)=D_{+}\left(\mu^{2}-E_{0}^{2}\right)-D_{-}\left(\mu^{2}-E_{0}^{2}\right) .
$$

Lemma 3.5. Let $\lambda \neq \lambda_{c}$, then $\delta:=\inf _{s \geq 0}\left|D_{ \pm}(s)\right|>0$.

Proof. If $\lambda=0$, then clearly $D_{ \pm}(s)=1>0$ for all $s \in[0, \infty)$. Let $\lambda \neq 0, \lambda_{c}$. Then $D_{ \pm}(0)=D(0) \neq 0$. Hence, by the continuity of $D_{ \pm}, D_{ \pm}$has no zeros near $s=0$. By the property that $\phi_{g}^{\prime}(x) \rightarrow 0$ as $x \rightarrow \infty$ and some estimate of $H \phi_{g}$, we can see that $\left(H \phi_{g}\right)(x) \rightarrow 0$ as $x \rightarrow \infty$. This fact implies that $\inf _{s_{0} \leq s} \operatorname{Re} D_{ \pm}(s)>0$ for a sufficiently large number $s_{0}>0$. In addition, $\operatorname{Im} D_{ \pm}(s)$ are positive for any closed interval included in $(0, \infty)$ by Assumption 2.7 (3) and the continuity of $\psi_{g}$. Hence we can see that $\inf _{s \geq 0}\left|D_{ \pm}(s)\right|>0$.

Remark 3.6. By Lemmas 3.3 and 3.5, we can see that there are constants $c, d, \varepsilon_{0}>0$ with $0<c<d$ and $\varepsilon_{0}>0$ such that

$$
c \leq\left|\frac{D(s \pm i \varepsilon)}{D_{ \pm}(s)}\right| \leq d
$$

for all $s \geq 0,0<\varepsilon<\varepsilon_{0}$. 


\subsection{Operators $R_{ \pm}$}

Lemma 3.7. One can define bounded operators $R_{ \pm}$on $\mathscr{H}$ as follows:

$$
R_{ \pm} f:=-\lambda \lim _{\varepsilon \downarrow 0} \int_{\left[E_{0}, \infty\right)} \frac{R_{\mu^{\prime 2} \pm i \varepsilon}\left(T^{2}\right) T^{1 / 2} g}{D_{ \pm}\left(\mu^{\prime 2}-E_{0}^{2}\right)} d\left\langle T^{1 / 2} g, E\left(\mu^{\prime}\right) f\right\rangle, \quad f \in \mathscr{H}
$$

where $R_{z}(A)$ is the resolvent of a linear operator $A$ at $z \in \rho(A)$ (the resolvent set of a linear operator $A)$.

Proof. For a fixed $\varepsilon>0$ and any $f \in \mathscr{H}$,

$$
\int_{\left[E_{0}, \infty\right)}\left\|\frac{R_{\mu^{\prime 2} \pm i \varepsilon}\left(T^{2}\right) T^{1 / 2} g}{D_{ \pm}\left(\mu^{\prime 2}-E_{0}^{2}\right)}\right\| d\left\|E\left(\mu^{\prime}\right) f\right\|^{2} \leq \frac{\|f\|^{2}\left\|T^{1 / 2} g\right\|}{\delta \varepsilon}<\infty
$$

by Lemma 3.5 and a property of a resolvent. Thus we can define linear operators $R_{ \pm}^{(\varepsilon)}$ on $\mathscr{H}$ by

$$
R_{ \pm}^{(\varepsilon)} f:=-\lambda \int_{\left[E_{0}, \infty\right)} \frac{R_{\mu^{\prime 2} \pm i \varepsilon}\left(T^{2}\right) T^{1 / 2} g}{D_{ \pm}\left(\mu^{\prime 2}-E_{0}^{2}\right)} d\left\langle T^{1 / 2} g, E\left(\mu^{\prime}\right) f\right\rangle
$$

in the sense of Bochner integral with the polarization identity. For any $h, f \in \mathscr{H}$,

$$
\begin{aligned}
& \left\langle h, R_{ \pm}^{(\varepsilon)} f\right\rangle \\
= & -\lambda \int_{\left[E_{0}, \infty\right)} \frac{\left\langle h, R_{\mu^{\prime 2} \pm i \varepsilon}\left(T^{2}\right) T^{1 / 2} g\right\rangle}{D_{ \pm}\left(\mu^{\prime 2}-E_{0}^{2}\right)} d\left\langle T^{1 / 2} g, E\left(\mu^{\prime}\right) f\right\rangle \\
= & -\lambda \int_{\left[E_{0}, \infty\right)} \int_{\left[E_{0}, \infty\right)} \frac{\mu^{1 / 2}}{\left(\mu^{2}-\mu^{\prime 2} \mp i \varepsilon\right) D_{ \pm}\left(\mu^{\prime 2}-E_{0}^{2}\right)} d\langle h, E(\mu) g\rangle d\left\langle T^{1 / 2} g, E\left(\mu^{\prime}\right) f\right\rangle,
\end{aligned}
$$

where we have used the functional calculus. By change of variables in the Lebesgue-Stieltjes integration, functional calculus and Fubini's theorem, we have

$$
\left\langle h, R_{ \pm}^{(\varepsilon)} f\right\rangle=\frac{\lambda \pi}{2} \int_{\left[E_{0}, \infty\right)}\left(A_{\varepsilon}^{(1)} * \phi_{g, f}^{ \pm}\right)\left(\mu^{2}\right) \mu^{1 / 2} \mp i\left(A_{\varepsilon}^{(2)} * \phi_{g, f}^{ \pm}\right)\left(\mu^{2}\right) \mu^{1 / 2} d\langle h, E(\mu) g\rangle
$$

where $\phi_{g, f}^{ \pm}(x)=\psi_{g, f}(\sqrt{x}) x^{-1 / 4} D_{ \pm}\left(x-E_{0}^{2}\right)^{-1} \chi_{\left[E_{0}^{2}, \infty\right)}(x), x \in \mathbb{R}$. We have $\phi_{g, f}^{ \pm} \in L^{2}(\mathbb{R})$ by Remark 2.8, and the function $\left(A_{\varepsilon}^{(j)} * \phi_{g, f}^{ \pm}\right)\left(\mu^{2}\right) \mu^{1 / 2}(\mu \in \mathbb{R})$ is in $L^{2}(\mathbb{R})$ for each $j=1,2$. Thus we have

$$
R_{ \pm}^{(\varepsilon)} f \rightarrow(\pi \lambda / 2)\left(H \phi_{g, f}^{ \pm}\right)\left(T^{2}\right) T^{1 / 2} g \mp(1 / 2) A_{ \pm} f \text { as } \varepsilon \downarrow 0
$$

by a property of Hilbert transform and the continuity of the inner product with $L^{2}(\mathbb{R})$, where the linear operators

$$
A_{ \pm} f:=i \pi \lambda \psi_{g, f}(T) D_{ \pm}\left(T^{2}-E_{0}^{2}\right)^{-1} g, f \in \mathscr{H}
$$


are well-defined (see Remark 2.8 and Lemma 3.5. Moreover, by change of variables, the isometry of Hilbert transform and Remark 2.8, we can show that the inequalities

$$
\left\|\left(H \phi_{g, f}^{ \pm}\right)\left(T^{2}\right) T^{1 / 2} g\right\| \leq \frac{c_{g}}{\delta}\|f\|, \quad\left\|A_{ \pm} f\right\| \leq \frac{2 \pi|\lambda| c_{g}}{\delta}\|f\|
$$

hold for all $f \in \mathscr{H}$ with constant $c_{g}:=\sup _{\sigma(T)} \psi_{g}$. Hence $R_{ \pm}$are bounded.

It is easy to see that $R_{ \pm}^{*}:=\left(R_{ \pm}\right)^{*}$ are given as follows: for $f \in \mathscr{H}$,

$$
\begin{aligned}
R_{ \pm}^{(\varepsilon) *} f & =\lambda \int_{\left[E_{0}, \infty\right)} R_{\mu^{\prime 2} \pm i \varepsilon}\left(T^{2}\right) D_{\mp}\left(T^{2}-E_{0}^{2}\right)^{-1} T^{1 / 2} g d\left\langle T^{1 / 2} g, E\left(\mu^{\prime}\right) f\right\rangle . \\
R_{ \pm}^{*} f & =\lim _{\varepsilon \downarrow 0} R_{ \pm}^{(\varepsilon) *} f .
\end{aligned}
$$

For a densely defined linear operator $A$ on a Hilbert space, we denote by $A^{\sharp} A$ or $A^{*}$.

Lemma 3.8. The ranges of $R_{ \pm}^{\sharp}$ are included in $D\left(T^{-1}\right) \cap D(T)$ and $R_{ \pm}^{\sharp}$ map $D(T)$ into $D\left(T^{2}\right)$.

Proof. For any $f, h \in \mathscr{H}$, we have

$$
\left\langle h, R_{ \pm} f\right\rangle=\frac{\lambda \pi}{2} \int_{\left[E_{0}, \infty\right)}\left(H \phi_{g, f}^{ \pm}\right)\left(\mu^{2}\right) \mu^{1 / 2} \mp i \frac{\psi_{g, f}(\mu)}{D_{ \pm}\left(\mu^{2}-E_{0}^{2}\right)} d\langle h, E(\mu) g\rangle .
$$

By change variable, we have

$$
\left(H \phi_{g, f}^{ \pm}\right)\left(\mu^{2}\right)=\left(H \psi_{T^{-1 / 2} g, f}^{ \pm}\right)(\mu)+\left(H \psi_{T^{-1 / 2} g, f}^{ \pm}\right)(-\mu), \mu \in \mathbb{R},
$$

where $\psi_{h, f}^{ \pm}(x):=\psi_{h, f}(x) D_{ \pm}\left(x^{2}-E_{0}\right)^{-1} \chi_{\left[E_{0}, \infty\right)}(x), x \in \mathbb{R}$ for $h, f \in \mathscr{H}$. Thus we see by Assumption 2.7 (3) and functional calculus that $\operatorname{Ran}\left(R_{ \pm}\right) \subset D\left(T^{-1}\right)$. The equation

$$
\mu\left(H \phi_{g, f}^{ \pm}\right)\left(\mu^{2}\right)=\left(H \psi_{T^{1 / 2} g, f}^{ \pm}\right)(\mu)-\left(H \psi_{T^{1 / 2} g, f}^{ \pm}\right)(-\mu), \mu \in \mathbb{R},
$$

operational calculus for (3.5) and Assumption 2.7 (3) imply that $\operatorname{Ran}\left(R_{ \pm}\right) \subset D(T)$. For any $f \in D(T)$ and $\mu \in \mathbb{R}$,

$$
\mu^{2}\left(H \phi_{g, f}^{ \pm}\right)\left(\mu^{2}\right)=\left(H \psi_{T^{1 / 2} g, T f}^{ \pm}\right)(\mu)+\left(H \psi_{T^{1 / 2} g, T f}^{ \pm}\right)(-\mu)-\frac{2}{\pi} \int_{\left[E_{0}, \infty\right)} \psi_{T^{1 / 2} g, f}^{ \pm}(x) d x .
$$

Hence $R_{ \pm} f \in D\left(T^{2}\right)$ and the following equation holds for any $h \in \mathscr{H}$

$$
\begin{aligned}
\left\langle h, T^{2} R_{ \pm} f\right\rangle= & \frac{\lambda \pi}{2} \int_{\left[E_{0}, \infty\right)}\left\{\left(H \psi_{T^{1 / 2} g, T f}^{ \pm}\right)(\mu)+\left(H \psi_{T^{1 / 2} g, T f}^{ \pm}\right)(-\mu)-\frac{2 c}{\pi}\right\} \mu^{1 / 2} d\langle h, E(\mu) g\rangle \\
& \mp i \frac{\lambda \pi}{2} \int_{\left[E_{0}, \infty\right)} \psi_{T^{1 / 2} g, T f}^{ \pm}(\mu) \mu^{1 / 2} d\langle h, E(\mu) g\rangle,
\end{aligned}
$$

where $c:=\int_{\mathbb{R}} \psi_{T^{1 / 2} g, f}^{ \pm}(x) d x$. In quite the same manner as in the case of $R_{ \pm}$, we can prove the statement for $R_{ \pm}^{*}$. 
Lemma 3.9. The operator equations $\left(R_{ \pm}\right)_{J}=R_{\mp}$ hold.

Proof. This follows from Assumption 2.7 (1) and Theorem 2.9.

Lemma 3.10. The operator equation $R_{-}=R_{+} \gamma+A_{-}$holds, where

$$
\gamma:=D_{+}\left(T^{2}-E_{0}^{2}\right) D_{-}\left(T^{2}-E_{0}^{2}\right)^{-1}
$$

is a bounded operator.

Proof. The first resolvent formula gives that, for any $\mu^{\prime}, \mu^{\prime \prime} \in \mathbb{R}, \varepsilon>0$,

$$
R_{\mu^{\prime 2}-i \varepsilon}\left(T^{2}\right)-R_{\mu^{\prime 2}+i \varepsilon}\left(T^{2}\right)=-2 i \varepsilon R_{\mu^{\prime 2}-i \varepsilon}\left(T^{2}\right) R_{\mu^{\prime 2}+i \varepsilon}\left(T^{2}\right) .
$$

Then, for any $f \in \mathscr{H}$,

$$
\begin{aligned}
R_{-}^{(\varepsilon)} f= & -\lambda \int_{\left[E_{0}, \infty\right)} \frac{R_{\mu^{\prime 2}+i \varepsilon}\left(T^{2}\right) T^{1 / 2} g}{D_{-}\left(\mu^{\prime 2}-E_{0}^{2}\right)} d\left\langle T^{1 / 2} g, E\left(\mu^{\prime}\right) f\right\rangle \\
& +2 i \lambda \varepsilon \int_{\left[E_{0}, \infty\right)} \frac{R_{\mu^{\prime 2}+i \varepsilon}\left(T^{2}\right) R_{\mu^{\prime 2}-i \varepsilon}\left(T^{2}\right) T^{1 / 2} g}{D_{-}\left(\mu^{\prime 2}-E_{0}^{2}\right)} d\left\langle T^{1 / 2} g, E\left(\mu^{\prime}\right) f\right\rangle .
\end{aligned}
$$

Thus, by change of variable, we have for any $h \in \mathscr{H}$

$$
\begin{aligned}
\left\langle h, R_{-}^{(\varepsilon)} f\right\rangle= & \left\langle h, R_{+}^{(\varepsilon)} \gamma f\right\rangle+2 i \lambda \int_{\left[E_{0}, \infty\right)} \int_{\left[E_{0}, \infty\right)} d\langle h, E(\mu) g\rangle d\left\langle T^{1 / 2} g, E\left(\mu^{\prime}\right) f\right\rangle \\
& \times \frac{\mu^{1 / 2} \varepsilon}{\left\{\left(\mu^{2}-\mu^{\prime 2}\right)^{2}+\varepsilon^{2}\right\} D_{-}\left(\mu^{\prime 2}-E_{0}^{2}\right)} \\
= & \left\langle h, R_{+}^{(\varepsilon)} \gamma f\right\rangle+i \pi \lambda \int_{\left[E_{0}, \infty\right)}\left(A_{\varepsilon}^{(2)} * \phi_{g, f}^{-}\right)\left(\mu^{2}\right) \mu^{1 / 2} d\langle h, E(\mu) g\rangle .
\end{aligned}
$$

By a property of the Poisson kernel, the function $\left(A_{\varepsilon}^{(2)} * \phi_{g, f}^{-}\right)\left(\mu^{2}\right) \mu^{1 / 2}(\mu \in \mathbb{R})$ converges to $\psi_{g, f}(\mu) / D_{-}\left(\mu^{2}-E_{0}^{2}\right)$ as $\varepsilon \rightarrow+0$ in the sense of $L^{2}(\mathbb{R})$. Hence the continuity of inner product with $L^{2}(\mathbb{R})$ implies that

$$
\begin{aligned}
\left\langle h, R_{-} f\right\rangle & =\left\langle h, R_{+} \gamma f\right\rangle+i \pi \lambda \int_{\left[E_{0}, \infty\right)} \frac{\psi_{g, f}(\mu)}{D_{-}\left(\mu^{2}-E_{0}^{2}\right)} d\langle h, E(\mu) g\rangle \\
& =\left\langle h, R_{+} \gamma f\right\rangle+\left\langle h, A_{-} f\right\rangle .
\end{aligned}
$$

Since $f$ and $h$ are arbitrary, one obtains the conclusion.

It is easy to see that

$$
\left(A_{-}\right)^{*}=-A_{+}
$$


Lemma 3.11. For any Borel measurable function $F$ on $\mathbb{R}, A_{ \pm} F(T) \subset F(T) A_{ \pm}$.

Proof. It is easy to see that for any $f \in D(F(T)), \psi_{g, F(T) f}=F \psi_{g, f} \in L^{2}(\mathbb{R})$. This fact and Lemma 3.5 imply that $\psi_{g, f}(T) g \in D(F(T))$ and $F(T) \psi_{g, f}(T) g=\psi_{g, F(T) f}(T) g$. Hence $A_{ \pm} f \in D(F(T))$ and $F(T) A_{ \pm} f=A_{ \pm} F(T) f$.

Lemma 3.12. The following operator equations hold:

$$
A_{-} R_{ \pm}^{*}=(\gamma-I) R_{ \pm}^{*}, \quad A_{-}\left(A_{-}\right)^{*}=-A_{-}-\left(A_{-}\right)^{*} .
$$

Proof. By applying Lemma 3.11 to the case $F=\chi_{B}$, one can easily see that $A_{ \pm} E(B)=$ $E(B) A_{ \pm}$holds for any $B \in \mathbf{B}^{1}$. For any $f, h \in \mathscr{H}$, we have

$$
\begin{aligned}
& \left\langle\left(A_{-}\right)^{*} h, R_{ \pm}^{(\varepsilon) *} f\right\rangle \\
= & i \pi \lambda^{2} \int_{\left[E_{0}, \infty\right)} \int_{\left[E_{0}, \infty\right)} \frac{\mu^{1 / 2} \psi_{g}(\mu)}{\left(\mu^{2}-\mu^{\prime 2} \mp i \varepsilon\right) D_{\mp}\left(\mu^{2}-E_{0}^{2}\right) D_{-}\left(\mu^{2}-E_{0}^{2}\right)} d\langle h, E(\mu) g\rangle d\left\langle T^{1 / 2} g, E\left(\mu^{\prime}\right) f\right\rangle .
\end{aligned}
$$

Then, since $\gamma$ and $E(B)$ commute on $\mathscr{H}$ for any $B \in \mathbf{B}^{1}$, (3.2) gives

$$
\begin{aligned}
& \left\langle\left(A_{-}\right)^{*} h, R_{ \pm}^{(\varepsilon) *} f\right\rangle \\
= & \lambda \int_{\left[E_{0}, \infty\right)} \int_{\left[E_{0}, \infty\right)} \frac{\mu^{1 / 2}}{\left(\mu^{2}-\mu^{\prime 2} \mp i \varepsilon\right) D_{\mp}\left(\mu^{2}-E_{0}^{2}\right)} d\langle h, E(\mu)(\gamma-1) g\rangle d\left\langle T^{1 / 2} g, E\left(\mu^{\prime}\right) f\right\rangle \\
= & \left\langle h,(\gamma-1) R_{ \pm}^{(\varepsilon) *} f\right\rangle .
\end{aligned}
$$

Thus, by a limit argument, we obtain $A_{-} R_{ \pm}^{*}=(\gamma-1) R_{ \pm}^{*}$. Moreover, 3.2 and the equation $\left(A_{-}\right)^{*}=-A_{+}$imply that

$$
\begin{aligned}
\left\langle h, A_{-}\left(A_{-}\right)^{*} f\right\rangle & =-(i \pi \lambda)^{2} \int_{\left[E_{0}, \infty\right)} \frac{\psi_{g, f}(\mu) \psi_{g}(\mu)}{D_{+}\left(\mu^{2}-E_{0}^{2}\right) D_{-}\left(\mu^{2}-E_{0}^{2}\right)} d\langle h, E(\mu) g\rangle \\
& =-i \pi \lambda \int_{\left[E_{0}, \infty\right)} \frac{\left(D_{+}\left(\mu^{2}-E_{0}^{2}\right)-D_{-}\left(\mu^{2}-E_{0}^{2}\right)\right) \psi_{g, f}(\mu)}{D_{+}\left(\mu^{2}-E_{0}^{2}\right) D_{-}\left(\mu^{2}-E_{0}^{2}\right)} d\langle h, E(\mu) g\rangle \\
& =-\left\langle h,\left(A_{-}\right)^{*} f+A_{-} f\right\rangle .
\end{aligned}
$$

Hence the equation $A_{-}\left(A_{-}\right)^{*}=-A_{-}-\left(A_{-}\right)^{*}$ holds.

\subsection{Operators $\Omega_{ \pm}$}

In this subsection we consider the bounded operators

$$
\Omega_{ \pm}:=I+R_{ \pm} .
$$


Let $x_{0}<0$ be the zero of $D(z)$ given in Lemma 3.1 (2) and

$$
U_{\mathrm{b}}:=\sqrt{\frac{\lambda}{D^{\prime}\left(x_{0}\right)}} R_{E_{0}^{2}+x_{0}}\left(T^{2}\right) T^{1 / 2} g, P:=\left\langle U_{\mathrm{b}}, \cdot\right\rangle U_{\mathrm{b}} .
$$

Then it is easy to see that $\left\|U_{\mathrm{b}}\right\|=1, U_{\mathrm{b}} \in D\left(T^{-1}\right) \cap D\left(T^{2}\right)$ and

$$
T U_{\mathrm{b}}=\sqrt{\lambda / D^{\prime}\left(x_{0}\right)} T^{-1 / 2} g+\left(E_{0}^{2}+x_{0}\right) T^{-1} U_{\mathrm{b}}
$$

Hence $P$ is a projection operator.

Lemma 3.13. Let $\lambda \neq \lambda_{\mathrm{c}}$. Then the following equations hold:

$$
\begin{aligned}
& \Omega_{ \pm}^{*} \Omega_{ \pm}=I \\
& \Omega_{ \pm} \Omega_{ \pm}^{*}=I-\theta\left(\lambda_{c}-\lambda\right) P
\end{aligned}
$$

where $\theta$ is the Heaviside function:

$$
\theta(t)= \begin{cases}1 & \text { if } \quad t>0 \\ 0 & \text { if } \quad t<0\end{cases}
$$

Remark 3.14. Lemma 3.13 implies that $\Omega_{ \pm}$are unitary operators if $\lambda>\lambda_{\mathrm{c}}$ and partial isometries with their final subspace $\operatorname{Ran}(I-P)$ if $\lambda<\lambda_{\mathrm{c}}$.

Proof. (1) We first prove (3.8).

It is sufficient to prove that $R_{ \pm}^{*} R_{ \pm}=-\left(R_{ \pm}+R_{ \pm}^{*}\right)$ hold. For any $f, h \in \mathscr{H}$ and $\varepsilon>0$,

$$
\begin{aligned}
\left\langle R_{ \pm}^{(\varepsilon)} h, R_{ \pm}^{(\varepsilon)} f\right\rangle= & \lambda^{2} \int_{\left[E_{0}, \infty\right)} \int_{\left[E_{0}, \infty\right)} d\left\langle h, E\left(\mu^{\prime}\right) T^{1 / 2} g\right\rangle d\left\langle T^{1 / 2} g, E\left(\mu^{\prime \prime}\right) f\right\rangle \\
& \times\left\langle\frac{R_{\mu^{\prime 2} \pm i \varepsilon}\left(T^{2}\right) T^{1 / 2} g}{D_{ \pm}\left(\mu^{\prime 2}-E_{0}^{2}\right)}, \frac{R_{\mu^{\prime \prime 2} \pm i \varepsilon}\left(T^{2}\right) T^{1 / 2} g}{D_{ \pm}\left(\mu^{\prime \prime 2}-E_{0}^{2}\right)}\right\rangle
\end{aligned}
$$

By the definition of the function $D$, we have

$$
\lambda\left\langle T^{1 / 2} g, R_{z}\left(T^{2}\right) T^{1 / 2} g\right\rangle=D\left(z-E_{0}^{2}\right)-1, z \in \mathbb{C} \backslash\left(E_{0}^{2}, \infty\right) .
$$

By this formula and a resolvent identity, we obtain

$$
\begin{aligned}
\left\langle R_{ \pm}^{(\varepsilon)} h, R_{ \pm}^{(\varepsilon)} f\right\rangle= & \lambda \int_{\left[E_{0}, \infty\right)} \int_{\left[E_{0}, \infty\right)} d\left\langle h, E\left(\mu^{\prime}\right) T^{1 / 2} g\right\rangle d\left\langle T^{1 / 2} g, E\left(\mu^{\prime \prime}\right) f\right\rangle \\
& \times \frac{D\left(\mu^{\prime 2}-E_{0}^{2} \mp i \varepsilon\right)-D\left(\mu^{\prime \prime 2}-E_{0}^{2} \pm i \varepsilon\right)}{\left(\mu^{\prime 2}-\mu^{\prime 2} \mp 2 i \varepsilon\right) D_{\mp}\left(\mu^{\prime 2}-E_{0}^{2}\right) D_{ \pm}\left(\mu^{\prime 2}-E_{0}^{2}\right)} \\
= & -\left\langle E_{ \pm}^{(\varepsilon)} h, R_{ \pm}^{(2 \varepsilon)} f\right\rangle-\left\langle R_{ \pm}^{(2 \varepsilon)} h, E_{ \pm}^{(\varepsilon)} f\right\rangle
\end{aligned}
$$


where the operators $E_{ \pm}^{(\varepsilon)}$ on $\mathscr{H}$ are given as follows:

$$
\left\langle h, E_{ \pm}^{(\varepsilon)} f\right\rangle:=\int_{\left[E_{0}, \infty\right)} \frac{D\left(\mu^{2}-E_{0}^{2} \pm i \varepsilon\right)}{D_{ \pm}\left(\mu^{2}-E_{0}^{2}\right)} d\langle h, E(\mu) f\rangle, \quad h, f \in \mathscr{H} .
$$

The inequality (3.3) implies that $E_{ \pm}^{(\varepsilon)}$ are bounded for all $0<\varepsilon<\varepsilon_{0}$. Thus, by the Lebesgue dominated convergence theorem, we have s- $\lim _{\varepsilon \downarrow 0} E_{ \pm}^{(\varepsilon)}=I$. Hence we obtain that $R_{ \pm}^{*} R_{ \pm}=-\left(R_{ \pm}+R_{ \pm}^{*}\right)$.

(2) We next prove (3.9) for $\lambda \neq \lambda_{c}$.

It is sufficient to prove that $R_{ \pm} R_{ \pm}^{*}=-\left(R_{ \pm}+R_{ \pm}^{*}\right)-\theta\left(\lambda_{c}-\lambda\right) P$ hold. For any $f, h \in \mathscr{H}$ and a fixed $\varepsilon>0,(3.4)$ implies

$$
\begin{aligned}
& \left\langle R_{ \pm}^{(\varepsilon) *} h, R_{ \pm}^{(\varepsilon) *} f\right\rangle \\
& =\lambda^{2} \int_{\left[E_{0}, \infty\right)} \int_{\left[E_{0}, \infty\right)} d\left\langle h, E(\mu) T^{1 / 2} g\right\rangle d\left\langle T^{1 / 2} g, E\left(\mu^{\prime}\right) f\right\rangle \\
& \times\left\langle R_{\mu^{2} \pm i \varepsilon}\left(T^{2}\right) D_{\mp}\left(T^{2}-E_{0}^{2}\right)^{-1} T^{1 / 2} g, R_{\mu^{\prime 2} \pm i \varepsilon}\left(T^{2}\right) D_{\mp}\left(T^{2}-E_{0}^{2}\right)^{-1} T^{1 / 2} g\right\rangle \\
& =\lambda^{2} \int_{\left[E_{0}, \infty\right)} \int_{\left[E_{0}, \infty\right)} \int_{\left[E_{0}, \infty\right)} d\left\langle h, E(\mu) T^{1 / 2} g\right\rangle d\left\langle T^{1 / 2} g, E\left(\mu^{\prime}\right) f\right\rangle d\left\|E\left(\mu^{\prime \prime}\right) g\right\|^{2} \\
& \times \frac{\mu^{\prime \prime}}{\left(\mu^{\prime \prime 2}-\mu^{2} \pm i \varepsilon\right)\left(\mu^{\prime \prime 2}-\mu^{\prime 2} \mp i \varepsilon\right) D_{ \pm}\left(\mu^{\prime \prime 2}-E_{0}^{2}\right) D_{\mp}\left(\mu^{\prime \prime 2}-E_{0}^{2}\right)} \\
& =\lambda \int_{\left[E_{0}, \infty\right)} \int_{\left[E_{0}, \infty\right)} \frac{1}{\mu^{2}-\mu^{\prime 2} \mp 2 i \varepsilon} J_{\varepsilon}^{ \pm}\left(\mu, \mu^{\prime}\right) d\left\langle h, E(\mu) T^{1 / 2} g\right\rangle d\left\langle T^{1 / 2} g, E\left(\mu^{\prime}\right) f\right\rangle \text {, }
\end{aligned}
$$

where, for any $\mu, \mu^{\prime} \in\left[E_{0}, \infty\right)$,

$$
\begin{aligned}
& J_{\varepsilon}^{ \pm}\left(\mu, \mu^{\prime}\right) \\
= & \int_{\left[E_{0}, \infty\right)} \frac{\lambda \mu^{\prime \prime}}{D_{ \pm}\left(\mu^{\prime \prime 2}-E_{0}^{2}\right) D_{\mp}\left(\mu^{\prime \prime 2}-E_{0}^{2}\right)}\left(\frac{1}{\mu^{\prime \prime 2}-\mu^{2} \pm i \varepsilon}-\frac{1}{\mu^{\prime 2}-\mu^{\prime 2} \mp i \varepsilon}\right) d\left\|E\left(\mu^{\prime \prime}\right) g\right\|^{2} .
\end{aligned}
$$

Then, by change of variable and $(3.2)$, one can show that

$$
J_{\varepsilon}^{ \pm}\left(\mu, \mu^{\prime}\right)=\lim _{R \rightarrow \infty} \frac{1}{2 \pi i} I_{\varepsilon, R}^{ \pm}\left(\mu, \mu^{\prime}\right),
$$

where, for $R>0$,

$$
I_{\varepsilon, R}^{ \pm}\left(\mu, \mu^{\prime}\right)=\int_{0}^{R}\left(\frac{1}{D_{+}(s)}-\frac{1}{D_{-}(s)}\right) G_{\mu, \mu^{\prime}}^{\varepsilon, \pm}(s) d s
$$

and

$$
G_{\mu, \mu^{\prime}}^{\varepsilon, \pm}(z):=\frac{1}{z-\mu^{\prime 2}+E_{0}^{2} \mp i \varepsilon}-\frac{1}{z-\mu^{2}+E_{0}^{2} \pm i \varepsilon}, z \in \mathbb{C}
$$


For $0<\eta<\varepsilon$ and $R>0$, let $C_{i}(i=1,2,3)$ be the curve given as follows:

$$
\begin{array}{ll}
C_{1}: \theta_{1}(t)=R-t-i \eta, & t: 0 \rightarrow R, \\
C_{2}: \theta_{2}(t)=\eta e^{-i t}, & t: \pi / 2 \rightarrow(3 \pi) / 2, \\
C_{3}: \theta_{3}(t)=t+i \eta, & t: 0 \rightarrow R .
\end{array}
$$

Then, for $C=C_{1}+C_{2}+C_{3}$, we have by the Lebesgue dominated convergence theorem,

$$
I_{\varepsilon, R}^{ \pm}\left(\mu, \mu^{\prime}\right)=\lim _{\eta \downarrow 0} \int_{C} \frac{1}{D(z)} G_{\mu, \mu^{\prime}}^{\varepsilon, \pm}(z) d z .
$$

We take $R$ such that $R>\max \left\{\mu^{2}-E_{0}^{2}, \mu^{\prime 2}-E_{0}^{2}\right\}$ and define a curve $C_{4}: \theta_{4}(t)=$ $\sqrt{\eta^{2}+R^{2}} e^{-i t}, t: t_{s} \rightarrow t_{f}$, for $t_{s}:=\arctan (\eta / R)$ and $t_{f}=2 \pi-t_{s}$. We consider two cases separately.

(i) The case $\lambda>\lambda_{\mathrm{c}}$. In this case, the function $G_{\mu, \mu^{\prime}}^{\varepsilon, \pm}(z) / D(z), z \in \mathbb{C} \backslash(0, \infty)$ has two simple poles at $z=\mu^{2}-E_{0}^{2} \mp i \varepsilon, z=\mu^{\prime 2}-E_{0}^{2} \pm i \varepsilon$. Then, by the residue theorem, we have

$$
\begin{aligned}
\int_{C} \frac{1}{D(z)} G_{\mu, \mu^{\prime}}^{\varepsilon, \pm}(z) d z= & 2 \pi i\left(\frac{1}{D\left(\mu^{\prime 2}-E_{0}^{2} \pm i \varepsilon\right)}-\frac{1}{D\left(\mu^{2}-E_{0}^{2} \mp i \varepsilon\right)}\right) \\
& -\int_{C_{4}} \frac{1}{D(z)} G_{\mu, \mu^{\prime}}^{\varepsilon, \pm}(z) d z .
\end{aligned}
$$

Thus, as $\eta$ tends to 0 , we have

$$
\begin{aligned}
I_{\varepsilon, R}^{ \pm}\left(\mu, \mu^{\prime}\right)= & 2 \pi i\left(\frac{1}{D\left(\mu^{\prime 2}-E_{0}^{2} \pm i \varepsilon\right)}-\frac{1}{D\left(\mu^{2}-E_{0}^{2} \mp i \varepsilon\right)}\right) \\
& -\lim _{\eta \downarrow 0} \int_{C_{4}} \frac{1}{D(z)} G_{\mu, \mu^{\prime}}^{\varepsilon, \pm}(z) d z .
\end{aligned}
$$

The definition of line integral implies

$$
\int_{C_{4}} \frac{1}{D(z)} G_{\mu, \mu^{\prime}}^{\varepsilon, \pm}(z) d z=-i \int_{t_{s}}^{2 \pi-t_{s}} \frac{G_{\mu, \mu^{\prime}}^{\varepsilon, \pm}\left(\sqrt{\eta^{2}+R^{2}} e^{-i t}\right) \sqrt{\eta^{2}+R^{2}} e^{-i t}}{D\left(\sqrt{\eta^{2}+R^{2}} e^{-i t}\right)} d t .
$$

By the triangle inequality, for any $t \in\left[t_{s}, t_{f}\right]$,

$$
\left|G_{\mu, \mu^{\prime}}^{\varepsilon, \pm}\left(\sqrt{\eta^{2}+R^{2}} e^{-i t}\right)\right| \leq \frac{\left|\mu^{2}-\mu^{\prime 2} \pm 2 i \varepsilon\right|}{\left(R-\left|\mu^{2}-E_{0}^{2} \pm i \varepsilon\right|\right)\left(R-\left|\mu^{\prime 2}-E_{0}^{2} \mp i \varepsilon\right|\right)} .
$$

On the other hand, by Lemma 3.5. (3.3) and the Lebesgue dominated convergence theorem, there are constants $\tilde{R}>0$ and $c_{0}>0$ such that $|D(z)| \geq c_{0}$ for all $|z| \geq \tilde{R}$. Thus we have

$$
I_{\varepsilon, R}^{ \pm}\left(\mu, \mu^{\prime}\right)=2 \pi i\left(\frac{1}{D\left(\mu^{\prime 2}-E_{0}^{2} \pm i \varepsilon\right)}-\frac{1}{D\left(\mu^{2}-E_{0}^{2} \mp i \varepsilon\right)}\right)+O\left(R^{-1}\right)(R \rightarrow \infty),
$$


where $O(\cdot)$ stands for the well known Landau symbol. Therefore we have

$$
J_{\varepsilon}^{ \pm}\left(\mu, \mu^{\prime}\right)=\frac{1}{D\left(\mu^{\prime 2}-E_{0}^{2} \pm i \varepsilon\right)}-\frac{1}{D\left(\mu^{2}-E_{0}^{2} \mp i \varepsilon\right)}
$$

for each $\mu, \mu^{\prime} \in\left[E_{0}, \infty\right)$. Thus, by 3.10$)$, we have

$$
\left\langle R_{ \pm}^{(\varepsilon) *} h, R_{ \pm}^{(\varepsilon) *} f\right\rangle=-\left\langle\left(R_{ \pm}^{(2 \varepsilon)}\right)^{*} h,\left(E_{ \pm}^{(\varepsilon)}\right)^{-1} f\right\rangle-\left\langle\left(E_{ \pm}^{(\varepsilon)}\right)^{-1} h,\left(R_{ \pm}^{(2 \varepsilon)}\right)^{*} f\right\rangle .
$$

As in the proof in (1), we obtain s- $\lim _{\varepsilon \downarrow 0}\left(E_{ \pm}^{(\varepsilon)}\right)^{-1}=I$. Therefore we obtain

$$
\lim _{\varepsilon \downarrow 0}\left\langle R_{ \pm}^{(\varepsilon) *} h, R_{ \pm}^{(\varepsilon) *} f\right\rangle=-\left\langle R_{ \pm}^{*} h, f\right\rangle-\left\langle h, R_{ \pm}^{*} f\right\rangle
$$

Thus we obtain the desired result.

(ii) The case $\lambda<\lambda_{\mathrm{c}}$. In this case, $G_{\mu, \mu^{\prime}}^{\varepsilon, \pm}(z) / D(z)$ has a simple pole at $z=x_{0}$ in addition to $z=\mu^{2}-E_{0}^{2} \mp i \varepsilon, z=\mu^{\prime 2}-E_{0}^{2} \pm i \varepsilon$. The residue $R_{0}$ of $G_{\mu, \mu^{\prime}}^{\varepsilon, \pm}(z) / D(z)$ at $z=x_{0}$ is give by

$$
R_{0}=\frac{1}{D^{\prime}\left(x_{0}\right)} \frac{\mu^{\prime 2}-\mu^{2} \pm 2 i \varepsilon}{\left(x_{0}-\mu^{\prime 2}+E_{0}^{2} \mp i \varepsilon\right)\left(x_{0}-\mu^{2}+E_{0}^{2} \pm i \varepsilon\right)} .
$$

Thus we have

$$
J_{\varepsilon}^{ \pm}\left(\mu, \mu^{\prime}\right)=\frac{1}{D\left(\mu^{\prime 2}-E_{0}^{2} \pm i \varepsilon\right)}-\frac{1}{D\left(\mu^{2}-E_{0}^{2} \mp i \varepsilon\right)}+R_{0}
$$

and also

$$
\frac{\lambda}{\mu^{2}-\mu^{\prime 2} \mp 2 i \varepsilon} R_{0}=-\frac{\lambda}{D^{\prime}\left(x_{0}\right)} \frac{1}{\left(\mu^{\prime 2}-E_{0}^{2}-x_{0} \pm i \varepsilon\right)\left(\mu^{2}-E_{0}^{2}-x_{0} \mp i \varepsilon\right)} .
$$

This implies that

$$
\begin{aligned}
& \lambda \lim _{\varepsilon \downarrow 0} \int_{\left[E_{0}, \infty\right)} \int_{\left[E_{0}, \infty\right)} \frac{1}{\mu^{2}-\mu^{\prime 2} \mp 2 i \varepsilon} R_{0} d\left\langle h, E(\mu) T^{1 / 2} g\right\rangle d\left\langle T^{1 / 2} g, E\left(\mu^{\prime}\right) f\right\rangle \\
= & -\left\langle h, U_{\mathrm{b}}\right\rangle\left\langle U_{\mathrm{b}}, f\right\rangle=-\langle h, P f\rangle,
\end{aligned}
$$

Thus we obtain the desired result. 


\subsection{Operators $U$ and $V$}

In this subsection, we investigate the operators $U$ and $V$ defined as follows:

$$
U:=\frac{1}{2}\left(T^{-1 / 2} \Omega_{+} T^{1 / 2}+T^{1 / 2} \Omega_{+} T^{-1 / 2}\right), V:=\frac{1}{2}\left(T^{-1 / 2} \Omega_{+} T^{1 / 2}-T^{1 / 2} \Omega_{+} T^{-1 / 2}\right),
$$

which are used to construct a Bogoliubov transformation. Then, by Lemma 3.8, one can easily see that $D(U)=D(V)=D\left(T^{-1 / 2}\right) \cap D\left(T^{1 / 2}\right)$.

Lemma 3.15. The operators $U$ and $V$ are bounded.

Proof. By (3.5) and Lemma 3.8 we have

$$
\begin{aligned}
\left\langle h, T^{-1 / 2} R_{ \pm} T^{1 / 2} f\right\rangle & =\frac{\lambda \pi}{2} \int_{\left[E_{0}, \infty\right)}\left(H \phi_{T^{1 / 2} g, f}^{ \pm}\right)\left(\mu^{2}\right) \mp i \frac{\psi_{g, f}(\mu)}{D_{ \pm}\left(\mu^{2}-E_{0}^{2}\right)} d\langle h, E(\mu) g\rangle, \\
\left\langle h, T^{1 / 2} R_{ \pm} T^{-1 / 2} f\right\rangle & =\frac{\lambda \pi}{2} \int_{\left[E_{0}, \infty\right)}\left(H \phi_{T^{-1 / 2} g, f}^{ \pm}\right)\left(\mu^{2}\right) \mu \mp i \frac{\psi_{g, f}(\mu)}{D_{ \pm}\left(\mu^{2}-E_{0}^{2}\right)} d\langle h, E(\mu) g\rangle .
\end{aligned}
$$

By Assumption 2.7 (3), (3.6), (3.7) and a property of Hilbert transform, we can show that

$$
\left\|T^{-1 / 2} R_{ \pm} T^{1 / 2} f\right\|,\left\|T^{1 / 2} R_{ \pm} T^{-1 / 2} f\right\| \leq \frac{|\lambda| \pi\left(C_{g}+c_{g}\right)}{2 \delta}\|f\|
$$

where $C_{g}:=\left(\sup _{E_{0}<x} x^{-1} \psi_{g}(x)\right)^{1 / 2}\left(\sup _{E_{0}<x} x \psi_{g}(x)\right)^{1 / 2}$. Hence the operators $T^{-1 / 2} R_{ \pm} T^{1 / 2}$ and $T^{1 / 2} R_{ \pm} T^{-1 / 2}$ are bounded.

In the same way as in the proof of Lemma 3.15 , we see that $T^{-1 / 2} R_{ \pm}^{*} T^{1 / 2}$ and $T^{1 / 2} R_{ \pm}^{*} T^{-1 / 2}$ are bounded on each domain $D\left(T^{1 / 2}\right)$ and $D\left(T^{-1 / 2}\right)$. In what follows, we write the bounded extension of $U$ and $V$ by the same symbol respectively. Then

$$
U^{*}=\frac{1}{2}\left(\overline{T^{-1 / 2} \Omega_{+}^{*} T^{1 / 2}}+\overline{T^{1 / 2} \Omega_{+}^{*} T^{-1 / 2}}\right) .
$$

Lemma 3.16. The operators $U^{\sharp}$ and $V^{\sharp}$ leave $D\left(T^{-1 / 2}\right)\left(\right.$ resp. $\left.D\left(T^{1 / 2}\right), D(T)\right)$ invariant. Proof. By applying Lemma 3.8 and using the equations

$$
U^{\sharp}=I+\frac{1}{2}\left(\overline{T^{-1 / 2} R_{+}^{\sharp} T^{1 / 2}}+\overline{T^{1 / 2} R_{+}^{\sharp} T^{-1 / 2}}\right),
$$

one can easily see that the assertion for $U^{\sharp}$ is true. Similarly one can prove the statement for $V^{\sharp}$.

Lemma 3.17. Let $F(x)=x^{ \pm 1 / 2}, x^{ \pm 1}$, a.e. $x \in(0, \infty)$. Then

$$
\Omega_{+} F(T) \Omega_{+}^{*}=\left(\Omega_{+}\right)_{J} F(T)\left(\Omega_{+}^{*}\right)_{J} \quad \text { on } D(F(T)) .
$$


Proof. By Lemma 3.8, the domain of each side of $(3.13)$ includes $D(F(T))$. By Lemmas 3.11 and 3.12 , we have

$$
\begin{aligned}
\left(\Omega_{+}\right)_{J} F(T)\left(\Omega_{+}^{*}\right)_{J}= & R_{+} F(T) R_{+}^{*}+R_{+}\left\{\left(A_{-}\right)^{*}+I\right\} F(T) \gamma+F(T) \gamma^{*}\left(A_{-}+I\right) R_{+}^{*} \\
& +F(T)\left\{A_{-}\left(A_{-}\right)^{*}+A_{-}+\left(A_{-}\right)^{*}+I\right\} \\
= & R_{+} F(T) R_{+}^{*}+R_{+} F(T)+F(T) R_{+}^{*}+F(T) \\
= & \Omega_{+} F(T) \Omega_{+}^{*} .
\end{aligned}
$$

\section{Commutation relations}

In this section, we prove that the pair $(U, V)$ satisfies the condition $(1.1), V$ is HilbertSchmidt and

$$
B(f):=A(U f)+A(J V f)^{*}, f \in \mathscr{H}
$$

satisfies some commutation relations with $H(\lambda)$. We denote the closure of $B(f)$ by the same symbol. By Lemma 3.16, we have $D\left(\mathrm{~d} \Gamma_{\mathrm{b}}(T)^{1 / 2}\right) \subset D(B(f)) \cap D\left(B(f)^{*}\right)$ for all $f \in D\left(T^{-1 / 2}\right)$.

Theorem 4.1. The following commutation relations hold:

(1) For any $f \in D(T)$ and $\psi \in \mathscr{F}_{\mathrm{b}, \text { fin }}(D(T))$,

$$
[H(\lambda), B(f)] \psi=-B(T f) \psi
$$

(2) For any $f \in D\left(T^{-1 / 2}\right) \cap D(T)$ and $\psi, \phi \in D\left(\mathrm{~d} \Gamma_{\mathrm{b}}(T)\right)$,

$$
\langle H(\lambda) \phi, B(f) \psi\rangle-\left\langle B(f)^{*} \phi, H(\lambda) \psi\right\rangle=-\langle\phi, B(T f) \psi\rangle .
$$

(3) For any $f \in D\left(T^{-1 / 2}\right) \cap D(T), B(f)$ maps $D\left(\mathrm{~d} \Gamma_{\mathrm{b}}(T)^{3 / 2}\right)$ into $D\left(\mathrm{~d} \Gamma_{\mathrm{b}}(T)\right)$ and for any $\psi \in D\left(\mathrm{~d} \Gamma_{\mathrm{b}}(T)^{3 / 2}\right)$,

$$
[H(\lambda), B(f)] \psi=-B(T f) \psi
$$

The both sides of $4.1,(4.2$ and 4.3 have meaning by Lemma 3.16. To prove this theorem, we prove the following lemma:

Lemma 4.2. For any $f \in D(T)$, the following equations hold:

$$
\begin{aligned}
& {[U, T] f=(V T+T V) f=} \frac{\lambda}{2}\left\langle D_{-}\left(T^{2}-E_{0}^{2}\right)^{-1} g, f\right\rangle g, \\
&\left(V^{*} J-U^{*}\right) g=-D_{-}\left(T^{2}-E_{0}^{2}\right)^{-1} g .
\end{aligned}
$$


Proof. For any $f, h \in D\left(T^{-1 / 2}\right) \cap D\left(T^{3 / 2}\right)$, we obtain

$$
\langle h,[U, T] f\rangle=\frac{1}{2}\left(\left\langle T^{1 / 2} R_{+}^{*} T^{-1 / 2} h, T f\right\rangle-\left\langle T h, T^{1 / 2} R_{+} T^{-1 / 2} f\right\rangle\right) .
$$

Then, for each $\varepsilon>0$, we have

$$
\begin{aligned}
& \left\langle T^{1 / 2} R_{ \pm}^{(\varepsilon) *} T^{-1 / 2} h, T f\right\rangle-\left\langle T h, T^{1 / 2} R_{ \pm}^{(\varepsilon)} T^{-1 / 2} f\right\rangle \\
= & \lambda \int_{\left[E_{0}, \infty\right)} \int_{\left[E_{0}, \infty\right)} \frac{\mu^{\prime 2}-\mu^{2}}{\left(\mu^{\prime 2}-\mu^{2} \pm i \varepsilon\right) D_{ \pm}\left(\mu^{\prime 2}-E_{0}^{2}\right)} d\langle h, E(\mu) g\rangle d\left\langle g, E\left(\mu^{\prime}\right) f\right\rangle \\
= & \lambda \int_{\left[E_{0}, \infty\right)} \int_{\left[E_{0}, \infty\right)} \frac{1}{D_{ \pm}\left(\mu^{\prime 2}-E_{0}^{2}\right)} d\langle h, E(\mu) g\rangle d\left\langle E\left(\mu^{\prime}\right) g, f\right\rangle \mp i \varepsilon\left\langle T^{-1 / 2} h, R_{ \pm}^{(\varepsilon)} T^{-1 / 2} f\right\rangle .
\end{aligned}
$$

Taking the limit $\varepsilon \downarrow 0$, we have

$$
\left\langle T^{1 / 2} R_{ \pm}^{*} T^{-1 / 2} h, T f\right\rangle-\left\langle T h, T^{1 / 2} R_{ \pm} T^{-1 / 2} f\right\rangle=\left\langle h, \lambda\left\langle D_{\mp}\left(T^{2}-E_{0}^{2}\right)^{-1} g, f\right\rangle g\right\rangle .
$$

Thus we have

$$
\langle h,[U, T] f\rangle=\frac{\lambda}{2}\left\langle h,\left\langle D_{-}\left(T^{2}-E_{0}^{2}\right)^{-1} g, f\right\rangle g\right\rangle .
$$

Since $D\left(T^{-1 / 2}\right) \cap D\left(T^{3 / 2}\right)$ is a core of $T$, the equation (4.4) holds for $f \in D(T)$. To prove (4.5), we note that

$$
\begin{aligned}
\left(V^{*} J-U^{*}\right) g & =\frac{1}{2}\left(T^{1 / 2} \Omega_{+}^{*} T^{-1 / 2} J-T^{-1 / 2} \Omega_{+}^{*} T^{1 / 2} J-T^{1 / 2} \Omega_{+}^{*} T^{-1 / 2}-T^{-1 / 2} \Omega_{+}^{*} T^{1 / 2}\right) g \\
& =-T^{-1 / 2} \Omega_{+}^{*} T^{1 / 2} g
\end{aligned}
$$

where we have used $J g=g$. Thus, for any $f \in \mathscr{H}$, we obtain

$$
\begin{aligned}
& \left\langle f,\left(V^{*} J-U^{*}\right) g\right\rangle \\
= & -\langle f, g\rangle-\lambda \lim _{\varepsilon \downarrow 0} \int_{\left[E_{0}, \infty\right)}\left\langle f, R_{\mu^{\prime 2}+i \varepsilon}\left(T^{2}\right) D_{-}\left(T^{2}-E_{0}^{2}\right)^{-1} g\right\rangle d\left\|E\left(\mu^{\prime}\right) T^{1 / 2} g\right\|^{2} \\
= & -\langle f, g\rangle+\lambda \lim _{\varepsilon \downarrow 0} \int_{\left[E_{0}, \infty\right)} \int_{\left[E_{0}, \infty\right)} \frac{\mu^{\prime}}{\mu^{\prime 2}-\mu^{2}+i \varepsilon} d\left\|E\left(\mu^{\prime}\right) g\right\|^{2} \frac{1}{D_{-}\left(\mu^{2}-E_{0}^{2}\right)}\langle f, E(\mu) g\rangle \\
= & -\langle f, g\rangle+\int_{\left[E_{0}, \infty\right)} \frac{D_{-}\left(\mu^{2}-E_{0}^{2}\right)-1}{D_{-}\left(\mu^{2}-E_{0}^{2}\right)} d\langle f, E(\mu) g\rangle \\
= & -\left\langle f, D_{-}\left(T^{2}-E_{0}^{2}\right)^{-1} g\right\rangle .
\end{aligned}
$$

Hence 4.5 holds.

Proof of Theorem 4.1. 
(1) By Lemma 3.16, for any $f \in D(T), B(f)$ leaves $\mathscr{F}_{\mathrm{b} \text {,fin }}(D(T))$ invariant and $H(\lambda)$ maps $\mathscr{F}_{\text {b,fin }}(D(T))$ into $\mathscr{F}_{\text {b,fin }}(\mathscr{H}) \subset D(B(f))$. Thus, by using (2.1), (9.3), we have for any $\psi \in \mathscr{F}_{\mathrm{b}, \mathrm{fin}}(D(T))$,

$$
[H(\lambda), B(f)] \psi=\left\{-A(T U f)+A(T J V f)^{*}-\frac{\lambda}{\sqrt{2}}\left\langle f,\left(V^{*} J-U^{*}\right) g\right\rangle \Phi_{\mathrm{s}}(g)\right\} \psi .
$$

Hence by Lemma 4.2, 4.1) holds.

(2) By Lemma 3.16 and fundamental properties of the annihilation operators and creation operators, we can see that, for any $f \in D\left(T^{-1 / 2}\right), D\left(\mathrm{~d} \Gamma_{\mathrm{b}}(T)^{1 / 2}\right) \subset D(B(f))$. For any $\psi, \phi \in D\left(\mathrm{~d} \Gamma_{\mathrm{b}}(T)\right)$, there are sequences $\psi_{n}, \phi_{n} \in \mathscr{F}_{\mathrm{b}, \text { fin }}(D(T)), n \in \mathbb{N}$ such that $\psi_{n} \rightarrow \psi, \phi_{n} \rightarrow \phi, \mathrm{d} \Gamma_{\mathrm{b}}(T) \psi_{n} \rightarrow \mathrm{d} \Gamma_{\mathrm{b}}(T) \psi, \mathrm{d} \Gamma_{\mathrm{b}}(T) \phi_{n} \rightarrow \mathrm{d} \Gamma_{\mathrm{b}}(T) \phi$ as $n \rightarrow \infty$, since $\mathscr{F}_{\mathrm{b}, \text { fin }}(D(T))$ is a core of $\mathrm{d} \Gamma_{\mathrm{b}}(T)$. By $(1)$, we have

$$
\left\langle H(\lambda) \phi_{n}, B(f) \psi_{k}\right\rangle-\left\langle B(f)^{*} \phi_{n}, H(\lambda) \psi_{k}\right\rangle=-\left\langle\phi_{n}, B(T f) \psi_{k}\right\rangle
$$

for all $n, k \in \mathbb{N}$ and $f \in D\left(T^{-1 / 2}\right) \cap D(T)$. By the fundamental inequalities (9.1) and (9.2) and the $\mathrm{d} \Gamma_{\mathrm{b}}(T)$-boundedness of $\Phi_{\mathrm{s}}(g)^{2}$, we can see that $\left\{B(f) \psi_{n}\right\}_{n=1}^{\infty},\left\{B(f) \phi_{n}\right\}_{n=1}^{\infty}$, $\left\{\Phi_{\mathrm{s}}(g)^{2} \psi_{n}\right\}_{n=1}^{\infty},\left\{\Phi_{\mathrm{s}}(g)^{2} \phi_{n}\right\}_{n=1}^{\infty}$ and $\left\{B(T f) \psi_{n}\right\}_{n=1}^{\infty}$ converge. Hence we obtain (4.2).

(3) By Lemma 3.16 and fundamental properties of the annihilation operators and creation operators, we see that, for any $f \in D\left(T^{-1 / 2}\right) \cap D(T), B(f) \operatorname{maps} D\left(\mathrm{~d} \Gamma_{\mathrm{b}}(T)^{3 / 2}\right)$ into $D\left(\mathrm{~d} \Gamma_{\mathrm{b}}(T)\right)$. Therefore, by 4.2 and the density of $D\left(\mathrm{~d} \Gamma_{\mathrm{b}}(T)\right)$, we have 4.3 .

\subsection{Relations of $U$ and $V$}

Lemma 4.3. Let $\lambda \neq \lambda_{\mathrm{c}}$. Then the following equations hold:

$$
\left\{\begin{aligned}
U^{*} U-V^{*} V & =I, \\
U_{J}^{*} V-V_{J}^{*} U & =0, \\
U U^{*}-V_{J} V_{J}^{*} & =I-\theta\left(\lambda_{c}-\lambda\right) Q_{+}, \\
U V^{*}-V_{J} U_{J}^{*} & =\theta\left(\lambda_{c}-\lambda\right) Q_{-},
\end{aligned}\right.
$$

where

$$
Q_{ \pm}:=\frac{1}{2}\left(\left\langle T^{1 / 2} U_{\mathrm{b}}, \cdot\right\rangle T^{-1 / 2} U_{\mathrm{b}} \pm\left\langle T^{-1 / 2} U_{\mathrm{b}}, \cdot\right\rangle T^{1 / 2} U_{\mathrm{b}}\right)
$$

are bounded operators on $\mathscr{H}$.

Proof. It is sufficient to prove (4.6) on $D\left(T^{-1 / 2}\right) \cap D\left(T^{1 / 2}\right)$. Using (3.8), one can show that the first equation in (3.8) hold. We have

$$
U_{J}^{*} V-V_{J}^{*} U=\frac{1}{2}\left(-T^{1 / 2}\left(\Omega_{+}^{*}\right)_{J} \Omega_{+} T^{-1 / 2}+T^{-1 / 2}\left(\Omega_{+}^{*}\right)_{J} \Omega_{+} T^{1 / 2}\right) .
$$


Multiplying the equation by $\left(\Omega_{+}\right)_{J}$ from the left, and using Lemma 3.17, we obtain

$$
\begin{aligned}
\left(\Omega_{+}\right)_{J}\left(U_{J}^{*} V-V_{J}^{*} U\right) & =\left(\Omega_{+}\right)_{J}\left(-T^{1 / 2}\left(\Omega_{+}^{*}\right)_{J} \Omega_{+} T^{-1 / 2}+T^{-1 / 2}\left(\Omega_{+}^{*}\right)_{J} \Omega_{+} T^{1 / 2}\right) \\
& =\Omega_{+}\left(-T^{1 / 2} \Omega_{+}^{*} \Omega_{+} T^{-1 / 2}+T^{-1 / 2} \Omega_{+}^{*} \Omega_{+} T^{1 / 2}\right)=0 .
\end{aligned}
$$

By (3.8), this implies that $U_{J}^{*} V-V_{J}^{*} U=0$. By Lemma 2.9 and Lemma 3.17, we have

$$
\begin{aligned}
V_{J} V_{J}^{*}= & \frac{1}{4}\left\{T^{-1 / 2}\left(\Omega_{+} T \Omega_{+}^{*}\right)_{J} T^{-1 / 2}-T^{-1 / 2}\left(\Omega_{+} \Omega_{+}^{*}\right)_{J} T^{1 / 2}\right. \\
& \left.\quad-T^{1 / 2}\left(\Omega_{+} \Omega_{+}^{*}\right)_{J} T^{-1 / 2}+T^{1 / 2}\left(\Omega_{+} T^{-1} \Omega_{+}^{*}\right)_{J} T^{1 / 2}\right\} \\
= & \frac{1}{4}\left(T^{-1 / 2} \Omega_{+} T \Omega_{+}^{*} T^{-1 / 2}-T^{-1 / 2} \Omega_{+} \Omega_{+}^{*} T^{1 / 2}\right. \\
& \left.\quad-T^{1 / 2} \Omega_{+} \Omega_{+}^{*} T^{-1 / 2}+T^{1 / 2} \Omega_{+} T^{-1} \Omega_{+}^{*} T^{1 / 2}\right) \\
= & V V^{*} .
\end{aligned}
$$

Hence, by direct calculations and (3.9), one obtains $U U^{*}-V_{J} V_{J}^{*}=I-\theta\left(\lambda_{c}-\lambda\right) Q_{+}$. Similarly one can prove the last equation in (4.6) (note that $P_{J}=P$ ).

\subsection{Hilbert-Schmidtness of $V$}

In this subsection, we show that $V$ is Hilbert-Schmidt. Then we can use Theorem 2.2 in the case of $\lambda>\lambda_{\mathrm{c}}$.

Lemma 4.4. The operator $V$ is Hilbert-Schmidt.

Proof. On $D\left(T^{-1 / 2}\right) \cap D\left(T^{1 / 2}\right), V^{*} V$ is calculated as follows:

$$
\begin{aligned}
V^{*} V= & \frac{1}{4}\left(T^{-1 / 2} R_{+} T^{1 / 2}+T^{1 / 2} R_{+}^{*} T^{-1 / 2}+T^{1 / 2}\left[R_{+}^{*}, T^{-1}\right] R_{+} T^{1 / 2}\right. \\
& +T^{1 / 2} R_{+} T^{-1 / 2}+T^{-1 / 2} R_{+}^{*} T^{1 / 2}+T^{-1 / 2}\left[R_{+}^{*}, T\right] R_{+} T^{-1 / 2} \\
& \left.\quad+T^{1 / 2} R_{+}^{*} R_{+} T^{-1 / 2}+T^{-1 / 2} R_{+}^{*} R_{+} T^{1 / 2}\right) \\
= & \frac{1}{4}\left(T^{1 / 2}\left[R_{+}^{*}, T^{-1}\right] R_{+} T^{1 / 2}+T^{-1 / 2}\left[R_{+}^{*}, T\right] R_{+} T^{-1 / 2}\right),
\end{aligned}
$$

where we have used the formula $R_{+}^{*} R_{+}=-\left(R_{+}+R_{+}^{*}\right)$ in the proof of Lemma 3.13 and Lemma 3.8. Thus, for any $f \in D\left(T^{-1 / 2}\right) \cap D\left(T^{1 / 2}\right)$ and $\varepsilon>0$, we have

$$
\begin{aligned}
& \left\langle f,\left(T^{1 / 2}\left[R_{+}^{(\varepsilon) *}, T^{-1}\right] R_{+}^{(\varepsilon)} T^{1 / 2}+T^{-1 / 2}\left[R_{+}^{(\varepsilon) *}, T\right] R_{+}^{(\varepsilon)} T^{-1 / 2}\right) f\right\rangle \\
= & \lambda \int_{\left[E_{0}, \infty\right)} \int_{\left[E_{0}, \infty\right)} \frac{\mu^{\prime}}{\left(\mu^{\prime 2}-\mu^{2}+i \varepsilon\right) D_{+}\left(\mu^{\prime 2}-E_{0}^{2}\right)} d\left\langle\left[T^{-1}, R_{+}^{(\varepsilon)}\right] T^{1 / 2} f, E(\mu) T^{1 / 2} g\right\rangle d\left\langle E\left(\mu^{\prime}\right) g, f\right\rangle \\
& +\lambda \int_{\left[E_{0}, \infty\right)} \int_{\left[E_{0}, \infty\right)} \frac{\mu}{\left(\mu^{\prime 2}-\mu^{2}+i \varepsilon\right) D_{+}\left(\mu^{\prime 2}-E_{0}^{2}\right)} d\left\langle\left[T, R_{+}^{(\varepsilon)}\right] T^{-1 / 2} f, E(\mu) T^{-1 / 2} g\right\rangle d\left\langle E\left(\mu^{\prime}\right) g, f\right\rangle .
\end{aligned}
$$


Then, for any $B \in \mathbf{B}^{1}$, we can see

$$
\begin{aligned}
& \left\langle\left[T^{-1}, R_{+}^{(\varepsilon)}\right] T^{1 / 2} f, E(B) T^{1 / 2} g\right\rangle \\
= & \lambda \int_{B} \int_{\left[E_{0}, \infty\right)} \frac{\mu^{\prime \prime}-\mu}{\left(\mu^{\prime \prime 2}-\mu^{2}-i \varepsilon\right) D_{-}\left(\mu^{\prime 2}-E_{0}^{2}\right)} d\left\langle f, E\left(\mu^{\prime \prime}\right) g\right\rangle d\|E(\mu) g\|^{2} .
\end{aligned}
$$

Similarly, we obtain

$$
\begin{aligned}
& \left\langle\left[T, R_{+}^{(\varepsilon)}\right] T^{-1 / 2} f, E(B) T^{-1 / 2} g\right\rangle \\
= & \lambda \int_{B} \int_{\left[E_{0}, \infty\right)} \frac{\mu-\mu^{\prime \prime}}{\left(\mu^{\prime \prime 2}-\mu^{2}-i \varepsilon\right) D_{-}\left(\mu^{\prime 2}-E_{0}^{2}\right)} d\left\langle f, E\left(\mu^{\prime \prime}\right) g\right\rangle d\|E(\mu) g\|^{2} .
\end{aligned}
$$

Thus, by a formula of change of variable in Lebesgue-Stieltjes integration and Fubini's theorem, we have

$$
\begin{aligned}
& \left\langle f,\left(T^{1 / 2}\left[R_{+}^{(\varepsilon) *}, T^{-1}\right] R_{+}^{(\varepsilon)} T^{1 / 2}+T^{-1 / 2}\left[R_{+}^{(\varepsilon) *}, T\right] R_{+}^{(\varepsilon)} T^{-1 / 2}\right) f\right\rangle \\
=\lambda^{2} & \int_{\left[E_{0}, \infty\right)} \int_{\left[E_{0}, \infty\right)} \int_{\left[E_{0}, \infty\right)} d\|E(\mu) g\|^{2} d\left\langle f, E\left(\mu^{\prime \prime}\right) g\right\rangle d\left\langle E\left(\mu^{\prime}\right) g, f\right\rangle \\
& \times \frac{\left(\mu-\mu^{\prime}\right)\left(\mu-\mu^{\prime \prime}\right)}{\left(\mu^{\prime 2}-\mu^{2}+i \varepsilon\right)\left(\mu^{\prime \prime 2}-\mu^{2}-i \varepsilon\right) D_{+}\left(\mu^{\prime 2}-E_{0}^{2}\right) D_{-}\left(\mu^{\prime \prime 2}-E_{0}^{2}\right)}
\end{aligned}
$$

Then it is easy to see that for any $\mu, \mu^{\prime}, \mu^{\prime \prime} \in\left[E_{0}, \infty\right)$

$$
\begin{aligned}
& \lim _{\varepsilon \downarrow 0} \frac{\left(\mu-\mu^{\prime}\right)\left(\mu-\mu^{\prime \prime}\right)}{\left(\mu^{\prime 2}-\mu^{2}+i \varepsilon\right)\left(\mu^{\prime \prime 2}-\mu^{2}-i \varepsilon\right) D_{+}\left(\mu^{\prime 2}-E_{0}^{2}\right) D_{-}\left(\mu^{\prime 2}-E_{0}^{2}\right)} \\
= & \frac{1}{\left(\mu^{\prime}+\mu\right)\left(\mu^{\prime \prime}+\mu\right) D_{+}\left(\mu^{\prime 2}-E_{0}^{2}\right) D_{-}\left(\mu^{\prime \prime 2}-E_{0}^{2}\right)} .
\end{aligned}
$$

For any $\varepsilon>0$ and $\mu, \mu^{\prime}, \mu^{\prime \prime} \in\left[E_{0}, \infty\right)$, we have by Lemma 3.5 and the arithmetic-geometric mean inequality

$$
\left|\frac{\left(\mu-\mu^{\prime}\right)\left(\mu-\mu^{\prime \prime}\right)}{\left(\mu^{\prime 2}-\mu^{2}+i \varepsilon\right)\left(\mu^{\prime 2}-\mu^{2}-i \varepsilon\right) D_{+}\left(\mu^{\prime 2}-E_{0}^{2}\right) D_{-}\left(\mu^{\prime \prime 2}-E_{0}^{2}\right)}\right| \leq \frac{1}{4 \delta^{2} \mu \sqrt{\mu^{\prime} \mu^{\prime \prime}}} .
$$

On the other side, for any $\alpha, \beta \in \mathbb{C}$, we see

$$
\begin{aligned}
& \int_{\left[E_{0}, \infty\right)} \int_{\left[E_{0}, \infty\right)} \int_{\left[E_{0}, \infty\right)} \frac{1}{\mu \sqrt{\mu^{\prime} \mu^{\prime \prime}}} d\|E(\mu) g\|^{2} d\left\|E\left(\mu^{\prime \prime}\right)(f+\alpha g)\right\|^{2} d\left\|E\left(\mu^{\prime}\right)(f+\beta g)\right\|^{2} \\
= & \left\|T^{-1 / 2} g\right\|^{2}\left\|T^{-1 / 4}(f+\alpha g)\right\|^{2}\left\|T^{-1 / 4}(f+\beta g)\right\|^{2}<\infty .
\end{aligned}
$$


Thus, by the Lebesgue dominated convergence theorem, we have

$$
\begin{gathered}
\lim _{\varepsilon \downarrow 0} \int_{\left[E_{0}, \infty\right)} \int_{\left[E_{0}, \infty\right)} \int_{\left[E_{0}, \infty\right)} d\|E(\mu) g\|^{2} d\left\|E\left(\mu^{\prime \prime}\right)(f+\alpha g)\right\|^{2} d\left\|E\left(\mu^{\prime}\right)(f+\beta g)\right\|^{2} \\
\times \frac{\left(\mu-\mu^{\prime}\right)\left(\mu-\mu^{\prime \prime}\right)}{\left(\mu^{\prime 2}-\mu^{2}+i \varepsilon\right)\left(\mu^{\prime \prime 2}-\mu^{2}-i \varepsilon\right) D_{+}\left(\mu^{\prime 2}-E_{0}^{2}\right) D_{-}\left(\mu^{\prime \prime 2}-E_{0}^{2}\right)} \\
=\int_{\left[E_{0}, \infty\right)} \int_{\left[E_{0}, \infty\right)} \int_{\left[E_{0}, \infty\right)} d\|E(\mu) g\|^{2} d\left\|E\left(\mu^{\prime \prime}\right)(f+\alpha g)\right\|^{2} d\left\|E\left(\mu^{\prime}\right)(f+\beta g)\right\|^{2} \\
\quad \times \frac{1}{\left(\mu^{\prime}+\mu\right)\left(\mu^{\prime \prime}+\mu\right) D_{+}\left(\mu^{\prime 2}-E_{0}^{2}\right) D_{-}\left(\mu^{\prime \prime 2}-E_{0}^{2}\right)} .
\end{gathered}
$$

In particular, for each $\alpha, \beta= \pm 1, \pm i$, the polarization identity and Fubini's theorem give

$$
\left\langle f, V^{*} V f\right\rangle=\frac{\lambda^{2}}{4} \int_{\left[E_{0}, \infty\right)}\left|\left\langle f, R_{-\mu}(T) D_{-}\left(T^{2}-E_{0}^{2}\right)^{-1} g\right\rangle\right|^{2} d\|E(\mu) g\|^{2} .
$$

Let $\left\{e_{n}\right\}_{n=1}^{\infty} \subset D\left(T^{-1 / 2}\right) \cap D\left(T^{1 / 2}\right)$ be a CONS of $\mathscr{H}$. The termwise integration implies that

$$
\begin{aligned}
\sum_{n=1}^{\infty}\left\langle e_{n}, V^{*} V e_{n}\right\rangle & =\frac{\lambda^{2}}{4} \int_{\left[E_{0}, \infty\right)}\left\|R_{-\mu}(T) D_{-}\left(T^{2}-E_{0}^{2}\right)^{-1} g\right\|^{2} d\|E(\mu) g\|^{2} \\
& =\frac{\lambda^{2}}{4} \int_{\left[E_{0}, \infty\right)} \int_{\left[E_{0}, \infty\right)} \frac{1}{\left(\mu^{\prime}+\mu\right)^{2}\left|D_{-}\left(\mu^{\prime 2}-E_{0}^{2}\right)\right|^{2}} d\left\|E\left(\mu^{\prime}\right) g\right\|^{2} d\|E(\mu) g\|^{2} \\
& \leq \frac{\lambda^{2}}{16 \delta^{2}} \int_{\left[E_{0}, \infty\right)} \int_{\left[E_{0}, \infty\right)} \frac{1}{\mu^{\prime} \mu} d\left\|E\left(\mu^{\prime}\right) g\right\|^{2} d\|E(\mu) g\|^{2}<\infty
\end{aligned}
$$

where we have used the arithmetic-geometric mean and Lemma 3.5. Hence $V$ is HilbertSchmidt.

Lemma 4.5. If $\lambda>\lambda_{\mathrm{c}}$, then there is a unitary operator $\mathbb{U}$ on $\mathscr{F}_{\mathrm{b}}(\mathscr{H})$ such that for all $f \in \mathscr{H}$,

$$
\mathbb{U} B(f) \mathbb{U}^{-1}=A(f)
$$

Proof. By Lemma 4.3 and Lemma 4.4, we can apply Theorem 2.2.

\section{Analysis in the case $\lambda>\lambda_{\mathrm{c}}$}

In this section we prove Theorem 2.10 (1). Before starting the proof, we need to know a property of the Hamiltonian $H(\lambda)$. 


\subsection{Time evolution}

Theorem 5.1 (Time evolution). If $\lambda>\lambda_{\mathrm{c}, 0}$, then for all $f \in D\left(T^{-1 / 2}\right), \psi \in D\left(\mathrm{~d} \Gamma_{\mathrm{b}}(T)^{1 / 2}\right)$ and $t \in \mathbb{R}$,

$$
\begin{aligned}
e^{i t H(\lambda)} B(f) e^{-i t H(\lambda)} \psi & =B\left(e^{i t T} f\right) \psi, \\
e^{i t H(\lambda)} B(f)^{*} e^{-i t H(\lambda)} \psi & =B\left(e^{i t T} f\right)^{*} \psi
\end{aligned}
$$

Proof. It is sufficient to prove (5.1), because (5.2) follows from taking the adjoint of (5.1). We define a function $v: \mathbb{R} \rightarrow \mathbb{C}$ by $v(t):=\left\langle\phi, e^{i t H(\lambda)} B\left(e^{-i t T} f\right) e^{-i t H(\lambda)} \psi\right\rangle, t \in \mathbb{R}$ for any $f \in D\left(T^{-1 / 2}\right) \cap D(T)$ and $\psi, \phi \in D\left(\mathrm{~d} \Gamma_{\mathrm{b}}(T)\right)$. Then $v$ is well-defined by operational calculus and Theorem 2.3. The function $v$ is differentiable and, by Theorem 4.1 (2), we have for any $t \in \mathbb{R}$

$$
\begin{aligned}
\frac{d}{d t} v(t)= & i\left\langle H(\lambda) e^{-i t H(\lambda)} \phi, B\left(e^{-i t T} f\right) e^{-i t H(\lambda)} \psi\right\rangle-i\left\langle B\left(e^{-i t T} f\right)^{*} e^{-i t H(\lambda)} \phi, H(\lambda) e^{-i t H(\lambda)} \psi\right\rangle \\
& +i\left\langle e^{-i t H(\lambda)} \phi, B\left(T e^{-i t T} f\right) e^{-i t H(\lambda)} \psi\right\rangle \\
= & 0 .
\end{aligned}
$$

Hence $v(t)=v(0)$ for all $t \in \mathbb{R}$. Hence the equation

$$
\left\langle\phi, e^{i t H(\lambda)} B\left(e^{-i t T} f\right) e^{-i t H(\lambda)} \psi\right\rangle=\langle\phi, B(f) \psi\rangle
$$

holds for all $t \in \mathbb{R}$. By replacing $f$ with $e^{i t T} f$, one has for all $\psi \in D\left(\mathrm{~d} \Gamma_{\mathrm{b}}(T)\right)$,

$$
e^{i t H(\lambda)} B(f) e^{-i t H(\lambda)} \psi=B\left(e^{i t T} f\right) \psi
$$

Since $D\left(\mathrm{~d} \Gamma_{\mathrm{b}}(T)\right)$ is a core of $(H(\lambda)+M)^{1 / 2}$ and $D(H(\lambda)+M)^{1 / 2}=D\left(\mathrm{~d} \Gamma_{\mathrm{b}}(T)^{1 / 2}\right)$ by Theorem 2.3 (3), we obtain (5.1) for $f \in D\left(T^{-1 / 2}\right) \cap D(T)$ and $\psi \in D\left(\mathrm{~d} \Gamma_{\mathrm{b}}(T)^{1 / 2}\right)$. Finally we extend (5.1) for all $f \in D\left(T^{-1 / 2}\right)$. Let $f \in D\left(T^{-1 / 2}\right)$ and $\psi \in D\left(\mathrm{~d} \Gamma_{\mathrm{b}}(T)^{1 / 2}\right)$. Then we set $f_{n}:=E((-\infty, n]) f$ for each $n \in \mathbb{N}$. Then $f_{n} \in D\left(T^{-1 / 2}\right) \cap D(T)$ for all $n \in \mathbb{N}$ and one can easily show that $f_{n} \rightarrow f, T^{-1 / 2} f_{n} \rightarrow T^{-1 / 2} f$ as $n \rightarrow \infty$ by using functional calculus and the the Lebesgue dominated convergence theorem. Thus we have $U f_{n} \rightarrow U f, J V f_{n} \rightarrow J V f$ as $n \rightarrow \infty$ by the boundedness of $U$ and $V$. By using the linearity of the Hilbert transform and that of the map $f \mapsto \psi_{g, f},(3.11)$ and (3.12), and (3.6), we can show that $T^{-1 / 2} U f_{n} \rightarrow$ $T^{-1 / 2} U f, T^{-1 / 2} J V f_{n} \rightarrow T^{-1 / 2} J V f$ as $n \rightarrow \infty$. Therefore we obtain $B\left(f_{n}\right) \phi \rightarrow B(f) \phi$ and $B\left(e^{i t T} f_{n}\right) \phi \rightarrow B\left(e^{i t T} f\right) \phi$ as $n \rightarrow \infty$ for any $\phi \in D\left(\mathrm{~d} \Gamma_{\mathrm{b}}(T)^{1 / 2}\right)$ by [3, Lemma4-28]. By the preceding result, we have for any $n \in \mathbb{N}$,

$$
B\left(f_{n}\right) e^{-i t H(\lambda)} \psi=e^{-i t H(\lambda)} B\left(e^{i t T} f_{n}\right) \psi
$$


The equation $D\left(\mathrm{~d} \Gamma_{\mathrm{b}}(T)^{1 / 2}\right)=D\left((H(\lambda)+M)^{1 / 2}\right)$ in Theorem 2.3 (3) implies that

$$
e^{-i t H(\lambda)} D\left(\mathrm{~d} \Gamma_{\mathrm{b}}(T)^{1 / 2}\right)=D\left(\mathrm{~d} \Gamma_{\mathrm{b}}(T)^{1 / 2}\right)
$$

Hence, taking the limit $n \rightarrow \infty$, we obtain (5.1) for $f \in D\left(T^{-1 / 2}\right), \psi \in D\left(\mathrm{~d} \Gamma_{\mathrm{b}}(T)^{1 / 2}\right)$.

\subsection{Proof of Theorem 2.10 (1)}

In this subsection, we assume that $\lambda>\lambda_{\mathrm{c}}$.

Lemma 5.2. Let $\Omega:=\mathbb{U}^{-1} \Omega_{0}$, where $\mathbb{U}$ is the unitary operator in Lemma 4.5 and $\Omega_{0}:=$ $(1,0,0, \ldots) \in \mathscr{F}_{\mathrm{b}}(\mathscr{H})$ is the Fock vacuum. Then there is an eigenvalue $E_{\mathrm{g}}$ of $H(\lambda)$ and $\Omega$ is a corresponding eigenvector: $H(\lambda) \Omega=E_{\mathrm{g}} \Omega$.

Proof. In general, by [3, Proposition4-4] for a dense subspace $\mathscr{D} \subset \mathscr{H}$, if $\psi \in \cap_{f \in \mathscr{D}} D(A(f))$ satisfies $A(f) \psi=0$ for all $f \in \mathscr{D}$, then there is a constant $\alpha \in \mathbb{C}$ such that $\psi=\alpha \Omega_{0}$. Thus, by Lemma 4.5, $B(f) \phi=0$ for all $f \in D\left(T^{-1 / 2}\right)$. Hence there is a constant $\alpha \in \mathbb{C}$ such that $\phi=\alpha \Omega$. For any $f \in D\left(T^{-1 / 2}\right)$ and $t \in \mathbb{R}$,

$$
B(f) e^{-i t H(\lambda)} \Omega=e^{-i t H(\lambda)} B\left(e^{i t T} f\right) \Omega=0
$$

by Lemma 5.1. Thus, for each $t \in \mathbb{R}$, there is a constant $\alpha(t) \in \mathbb{C}$ such that $e^{-i t H(\lambda)} \Omega=\alpha(t) \Omega$. Then we have $|\alpha(t)|=1, \alpha(t+s)=\alpha(t) \alpha(s)$ for all $t, s \in \mathbb{R}$, since $\left\{e^{-i t H(\lambda)}\right\}_{t \in \mathbb{R}}$ is a strongly continuous one-parameter unitary group. Thus there exists a constant $E_{\mathrm{g}} \in \mathbb{R}$ such that $\alpha(t)=e^{-i t E_{\mathrm{g}}}, t \in \mathbb{R}$. The differentiation of the equation $e^{-i t H(\lambda)} \Omega=e^{-i t E_{\mathrm{g}}} \Omega$ in $t$ implies $\Omega \in D(H(\lambda))$ and $\Omega \in \operatorname{Ker}\left(H(\lambda)-E_{\mathrm{g}}\right)$.

Proof of Theorem 2.10 (1).

The subspace $\mathcal{U}:=\mathscr{L}\left\{B\left(f_{1}\right)^{*} \cdots B\left(f_{n}\right)^{*} \Omega, \Omega \mid f_{j} \in D\left(T^{-1 / 2}\right), j=1, \ldots, n, n \in \mathbb{N}\right\}$ is dense in $\mathscr{F}_{\mathrm{b}}(\mathscr{H})$ by the fact that $\mathcal{U}=\mathbb{U}^{-1} \mathscr{F}_{\mathrm{b}, \text { fin }}\left(D\left(T^{-1 / 2}\right)\right)$, where $\mathscr{L}(\mathscr{D})$ denotes the subspace algebraically spanned by the vectors in $\mathscr{D}$. By Lemma 5.1 and Lemma 9.3, for any $t \in$ $\mathbb{R}, f_{j} \in D\left(T^{-1 / 2}\right), j=1, \ldots, n$, we have

$$
\begin{aligned}
e^{i t H(\lambda)} B\left(f_{1}\right)^{*} \cdots B\left(f_{n}\right)^{*} \Omega & =B\left(e^{i t T} f_{1}\right)^{*} \cdots B\left(e^{i t T} f_{n}\right)^{*} e^{i t H(\lambda)} \Omega \\
& =B\left(e^{i t T} f_{1}\right)^{*} \cdots B\left(e^{i t T} f_{n}\right)^{*} e^{i t E_{\mathrm{g}}} \Omega \\
& =e^{i t E_{\mathrm{g}}} \mathbb{U}^{-1} e^{i t \mathrm{~d} \Gamma_{\mathrm{b}}(T)} A\left(f_{1}\right)^{*} \cdots A\left(f_{n}\right)^{*} \Omega_{0} \\
& =\mathbb{U}^{-1} e^{i t\left(\mathrm{~d} \Gamma_{\mathrm{b}}(T)+E_{\mathrm{g}}\right)} \mathbb{U} B\left(f_{1}\right)^{*} \cdots B\left(f_{n}\right)^{*} \Omega .
\end{aligned}
$$


By this equation and a limiting argument, we obtain $\mathbb{U} e^{i t H(\lambda)} \mathbb{U}^{-1}=e^{i t\left(\mathrm{~d} \Gamma_{\mathrm{b}}(T)+E_{\mathrm{g}}\right)}$. By the unitary covariance of functional calculus, we have

$$
\mathbb{U} e^{i t H(\lambda)} \mathbb{U}^{-1}=e^{i t \mathbb{U} H(\lambda) \mathbb{U}^{-1}}, \quad t \in \mathbb{R} .
$$

Hence (2.7) holds. The equation (2.7) and the well-known spectral properties of $\mathrm{d} \Gamma_{\mathrm{b}}(T)$ imply that $E_{\mathrm{g}}$ is the ground state energy of $H(\lambda)$ and $\Omega$ is the unique ground state of $H(\lambda)$.

Lemma 5.3. The ground state energy $E_{\mathrm{g}}$ is given as follows:

$$
\begin{aligned}
& E_{\mathrm{g}}=\frac{\lambda}{4}\|g\|^{2}-\operatorname{Tr}\left(T^{1 / 2} V^{*} V T^{1 / 2}\right) \\
& \operatorname{Tr}\left(T^{1 / 2} V^{*} V T^{1 / 2}\right)=\frac{\lambda^{2}}{4} \int_{\left[E_{0}, \infty\right)} \int_{\left[E_{0}, \infty\right)} \frac{\mu}{\left(\mu+\mu^{\prime}\right)^{2}\left|D_{-}\left(\mu^{2}-E_{0}^{2}\right)\right|^{2}} d\|E(\mu) g\|^{2} d\left\|E\left(\mu^{\prime}\right) g\right\|^{2} .
\end{aligned}
$$

Proof. The operator $\mathbb{U}$ leaves $D\left(\mathrm{~d} \Gamma_{\mathrm{b}}(T)\right)$ invariant by Theorem $2.10(1)$. In particular, $\mathbb{U} \Omega_{0} \in$ $D\left(\mathrm{~d} \Gamma_{\mathrm{b}}(T)^{1 / 2}\right)$. Thus, by Lemma 9.4 , the isometry of $\mathbb{U}$ and the definition of $B(\cdot)$, we have $\left\langle\Omega_{0},\left(H(\lambda)-E_{\mathrm{g}}\right) \Omega_{0}\right\rangle=\operatorname{Tr}\left(T^{1 / 2} V^{*} V T^{1 / 2}\right)$. It is easy to see that $\left\langle\Omega_{0}, H(\lambda) \Omega_{0}\right\rangle=\lambda\|g\|^{2} / 4$. Hence (5.3) holds. Formula $(5.4)$ can be proved in the same way as in 4.8.

\section{Analysis in the case $\lambda_{\mathrm{c}, 0}<\lambda<\lambda_{\mathrm{c}}$}

In Section 5, we proved Theorem 2.10 (1). But the proof is valid only for the case $\lambda>\lambda_{c}$. Therefore it is necessary to find another pair of operators $U$ and $V$ if one wants to use a Bogoliubov transformation for the spectral analysis of $H(\lambda)$ in the case $\lambda \leq \lambda_{\mathrm{c}}$. In this section we assume that $T$ and $g$ satisfy Assumption 2.7, $E_{0}>0$ and $\lambda_{\mathrm{c}, 0}<\lambda<\lambda_{\mathrm{c}}$. Under these conditions, we can define operators $\xi, X, Y$ and $T_{ \pm}$as follows:

$$
\begin{aligned}
& \xi:=\Omega_{+} T \Omega_{+}^{*}+\beta P, \\
& X:=U \Omega_{+}^{*}+T_{+} P, Y:=V \Omega_{+}^{*}+T_{-} P, \\
& T_{ \pm}:=\frac{1}{2}\left(\beta^{1 / 2} T^{-1 / 2} \pm \beta^{-1 / 2} T^{1 / 2}\right),
\end{aligned}
$$

where $\beta:=\left(E_{0}^{2}+x_{0}\right)^{1 / 2}$.

Remark 6.1. The definition of $x_{0}$ implies the following:

$$
E_{0}^{2}+x_{0}\left\{\begin{array}{ccc}
>0, & \text { if } & \lambda_{\mathrm{c}, 0}<\lambda<\lambda_{\mathrm{c}} \\
=0, & \text { if } & \lambda=\lambda_{\mathrm{c}, 0} \\
<0, & \text { if } & \lambda<\lambda_{\mathrm{c}, 0}
\end{array}\right.
$$


Thus, in the case $\lambda_{c, 0}<\lambda<\lambda_{\mathrm{c}}$, we see that the inequality $0<\beta<E_{0}$ holds. Let

$$
C(f):=A(X f)+A(J Y f)^{*}, f \in \mathscr{H} .
$$

Then $C(f)$ is a densely defined closable operator. We denotes its closure by the same symbol.

\subsection{Properties of $X, Y$ and $\xi$}

In this subsection we study operators $X, Y$ and $\xi$. First, we consider $\xi$. Let be

$$
\tilde{T}:=\Omega_{+} T \Omega_{+}^{*} .
$$

Lemma 6.2. The operator $\tilde{T}$ is a self-adjoint operator with $D(\tilde{T})=D(T)$.

Proof. By Lemma 3.8 we see that $D(\tilde{T})=D(T)$. Hence $\tilde{T}$ is symmetric. For any $\phi \in$ $D\left((\tilde{T})^{*}\right)$ and $\psi \in D(T)=D(\tilde{T})$, we have $\left\langle\Omega_{+}^{*}(\tilde{T})^{*} \phi, \psi\right\rangle=\left\langle\Omega_{+}^{*} \phi, T \psi\right\rangle$. This implies that $\Omega_{+}^{*} \phi \in D(T)$. Hence $\tilde{T}$ is self-adjoint.

Lemma 6.3. The spectra of $\tilde{T}$ are as follows:

$$
\sigma(\tilde{T})=\{0\} \cup \sigma(T), \sigma_{\mathrm{ac}}(\tilde{T})=\sigma(T), \sigma_{\mathrm{p}}(\tilde{T})=\{0\}, \sigma_{\mathrm{sc}}(\tilde{T})=\emptyset .
$$

Proof. We define a family of projection operators $\left\{E_{P}(B) \mid B \in \mathbf{B}^{1}\right\}$ on $\mathscr{H}$ as follows: $E_{P}(B)=0$ if $0 \notin B$ and $E_{P}(B)=P$ if $0 \in B$ for each $B \in \mathbf{B}^{1}$. It is easy to see that $\left\{E_{\tilde{T}}(B):=\Omega_{+} E(B) \Omega_{+}^{*}+E_{P}(B) \mid B \in \mathbf{B}^{1}\right\}$ is a spectral measure. Using functional calculus, we see that $E_{\tilde{T}}(\cdot)$ is the spectral measure of $\tilde{T}$. It is easy to see that the absolutely continuous part (resp. singular part) of $\tilde{T}$ is $\tilde{T} \uparrow \operatorname{Ran}(I-P)(\operatorname{resp} . \tilde{T}\lceil\operatorname{Ran}(P))$ since $T$ is absolutely continuous and $\Omega_{ \pm}$are partial isometries. Thus we see $\sigma(\tilde{T})=\{0\} \cup \sigma_{\mathrm{ac}}(\tilde{T}), \sigma_{\mathrm{p}}(\tilde{T})=$ $\{0\}, \sigma_{\mathrm{sc}}(\tilde{T})=\emptyset$.

We next show that $\sigma_{\mathrm{ac}}(\tilde{T})=\sigma(T)$. For any $\mu \in \sigma(T)$, there is a sequence $\psi_{n} \in D(T), n \in$ $\mathbb{N}$ such that $\left\|\psi_{n}\right\|=1$ for all $n \in \mathbb{N}$ and $\lim _{n \rightarrow \infty}\left\|(T-\mu) \psi_{n}\right\|=0$. For each $n \in \mathbb{N}$, there is a $\phi_{n} \in \operatorname{Ran}(I-P)$ such that $\psi_{n}=\Omega_{+}^{*} \phi_{n}$. Then $\left\|\phi_{n}\right\|=\left\|\Omega_{+} \psi_{n}\right\|=\left\|\psi_{n}\right\|=1$ and $\left\|(\tilde{T}-\mu) \phi_{n}\right\|=\left\|(T-\mu) \psi_{n}\right\| \rightarrow 0$ as $n \rightarrow \infty$. Thus we have $\mu \in \sigma(\tilde{T} \nmid \operatorname{Ran}(I-P))=\sigma_{\mathrm{ac}}(\tilde{T})$. For any $\mu \in \sigma_{\text {ac }}(\tilde{T})$, there is a sequence $\eta_{n} \in D(\tilde{T}) \cap \operatorname{Ran}(I-P)$ such that $\left\|\eta_{n}\right\|=1$ and $\lim _{n \rightarrow \infty}\left\|(\tilde{T}-\mu) \eta_{n}\right\|=0$. Then we easily see that $\Omega_{+}^{*} \eta_{n} \in D(T)$ for all $n \in \mathbb{N}$. The equation $\Omega_{+} \Omega_{+}^{*} \eta_{n}=\eta_{n}$ implies that $\left\|\Omega_{+}^{*} \eta_{n}\right\|=1$ for all $n \in \mathbb{N}$ and

$$
\left\|(T-\mu) \Omega_{+}^{*} \eta_{n}\right\|=\left\|(\tilde{T}-\mu) \eta_{n}\right\| \rightarrow 0, \quad n \rightarrow \infty .
$$

Thus $\mu \in \sigma(T)$. Hence $\sigma_{\text {ac }}(\tilde{T})=\sigma(T)$. 
Lemma 6.4. The operator $\xi$ is an injective, non-negative self-adjoint operator with $D(\xi)=$ $D(T)$ and we have the following equations:

$$
\sigma(\xi)=\{\beta\} \cup \sigma(T), \sigma_{\mathrm{ac}}(\xi)=\sigma(T), \sigma_{\mathrm{p}}(\xi)=\{\beta\}, \sigma_{\mathrm{sc}}(\xi)=\emptyset
$$

In particular, $\beta$ is the ground state energy of $\xi$, which is an isolated eigenvalue of $\xi$, and $U_{\mathrm{b}}$ is the unique ground state of $\xi$.

Proof. By Lemma 6.3 and a spectral property of direct sum of self-adjoint operators, we have the equation 6.1. Thus $\beta$ is an isolated ground state energy by Remark 6.1. It is easy to see that $U_{\mathrm{b}}$ is a ground state of $\xi$. Assume that $f \in \operatorname{Ker}(\xi-\beta)$ satisfies $(I-P) f \neq 0$. Then $\Omega_{+}^{*} f \neq 0$ by Lemma 3.13 . This implies $T \Omega_{+}^{*} f=\beta \Omega_{+}^{*} f$, but this contradicts Assumption 2.7 (1). Hence $(I-P) f=0$, implying that the ground state of $\xi$ is unique.

Lemma 6.5. The operators $\xi^{ \pm 1 / 2}$ are given by

$$
\begin{gathered}
\xi^{1 / 2}=\Omega_{+} T^{1 / 2} \Omega_{+}^{*}+\beta^{1 / 2} P \\
\xi^{-1 / 2}=\Omega_{+} T^{-1 / 2} \Omega_{+}^{*}+\beta^{-1 / 2} P
\end{gathered}
$$

with $D\left(\xi^{ \pm 1 / 2}\right)=D\left(T^{ \pm 1 / 2}\right)$.

Proof. We can show in the same way as in the proof of Lemma 6.4 that the right hand side of 6.2 is non-negative, self-adjoint operator with its domain $D\left(T^{1 / 2}\right)$. We have $\xi \subset$ $\left(\Omega_{+} T^{1 / 2} \Omega_{+}^{*}+\beta^{1 / 2} P\right)^{2}$. Since a self-adjoint operator has no non-trivial symmetric extension, (6.2) holds. In the same way as in the case of 6.2), we can show that the right hand side of 6.3$)$ is a self-adjoint operator. We have $D\left(\Omega_{+} T^{-1 / 2} \Omega_{+}^{*}+\beta^{-1 / 2} P\right) \subset \operatorname{Ran}\left(\xi^{1 / 2}\right)$ and $\xi^{1 / 2}\left(\Omega_{+} T^{-1 / 2} \Omega_{+}^{*}+\beta^{-1 / 2} P\right)=I$ on $D\left(\Omega_{+} T^{-1 / 2} \Omega_{+}^{*}\right)$. Hence $\Omega_{+} T^{-1 / 2} \Omega_{+}^{*}+\beta^{-1 / 2} P \subset \xi^{-1 / 2}$. Thus the equation 6.3 holds.

Next, we study $X$ and $Y$.

Lemma 6.6. The operators $X^{\sharp}$ and $Y^{\sharp}$ leave $D\left(T^{-1 / 2}\right)$ (resp. $\left.D\left(T^{1 / 2}\right), D(T)\right)$ invariant.

Proof. The assertion follows from Lemma 3.8, Lemma 3.16, Lemma 6.5 and the definition of $X$ and $Y$.

Lemma 6.7. The following equations hold:

$$
\left\{\begin{aligned}
X^{*} X-Y^{*} Y & =I, \\
X_{J}^{*} Y-Y_{J}^{*} X & =0, \\
X X^{*}-Y_{J} Y_{J}^{*} & =I, \\
X Y^{*}-Y_{J} X_{J}^{*} & =0 .
\end{aligned}\right.
$$


Proof. The operator $P$ (resp. $T_{ \pm}$) satisfies $P_{J}=P\left(\operatorname{resp.}\left(T_{ \pm}\right)_{J}=T_{ \pm}\right)$. By (3.9), we have $\Omega_{+}^{*} U_{\mathrm{b}}=0$. Hence we obtain $\left(U^{*} \pm V^{*}\right) T^{ \pm 1 / 2} U_{\mathrm{b}}=0$ and $\left(U^{*} T_{ \pm}-V^{*} T_{\mp}\right) U_{\mathrm{b}}=0$. The equations $T_{+} T_{+}-T_{-} T_{-}=I$ and $T_{+} T_{-}-T_{-} T_{+}=0$ hold on $D\left(T^{-1}\right) \cap D(T)$. By 4.6 and direct calculations, we have $X^{*} X-Y^{*} Y=I$ and $X_{J}^{*} Y-Y_{J}^{*} X=0$. By similar calculations, we have $X X^{*}-Y_{J} Y_{J}^{*}=I$ and $X Y^{*}-Y_{J} X_{J}^{*}=0$ on $D\left(T^{-1 / 2}\right) \cap D\left(T^{1 / 2}\right)$. Then, by a limiting argument, we obtain 6.4).

Lemma 6.8. The operator $Y$ is Hilbert-Schmidt.

Proof. We can easily show that the assertion follows from Lemma 4.4, Lemma 6.6 and the choice a CONS $\left\{e_{n}\right\}_{n=0}^{\infty} \subset D\left(T^{-1 / 2}\right) \cap D\left(T^{1 / 2}\right)$ with $e_{0}=U_{\mathrm{b}}$.

Lemma 6.9. There is a unitary operator $\mathbb{V}$ on $\mathscr{F}_{\mathrm{b}}(\mathscr{H})$ such that for all $f \in \mathscr{H}$,

$$
\mathbb{V} C(f) \mathbb{V}^{-1}=A(f)
$$

Proof. By Theorem 2.2, (6.4) and Lemma 6.8, we can prove this assertion.

\subsection{Commutation relations}

Theorem 6.10. The following commutation relations hold:

(1) For any $f \in D(T)$ and $\psi \in \mathscr{F}_{\mathrm{b}, \mathrm{fin}}(D(T))$,

$$
[H(\lambda), C(f)] \psi=-C(\xi f) \psi
$$

(2) For any $f \in D\left(T^{-1 / 2}\right) \cap D(T)$ and $\psi, \phi \in D\left(\mathrm{~d} \Gamma_{\mathrm{b}}(T)\right)$,

$$
\langle H(\lambda) \phi, C(f) \psi\rangle-\left\langle C(f)^{*} \phi, H(\lambda) \psi\right\rangle=-\langle\phi, C(\xi f) \psi\rangle .
$$

(3) For any $f \in D\left(T^{-1 / 2}\right) \cap D(T), C(f)$ maps $D\left(\mathrm{~d} \Gamma_{\mathrm{b}}(T)^{3 / 2}\right)$ into $D\left(\mathrm{~d} \Gamma_{\mathrm{b}}(T)\right)$ and for any $\psi \in D\left(\mathrm{~d} \Gamma_{\mathrm{b}}(T)^{3 / 2}\right)$,

$$
[H(\lambda), C(f)] \psi=-C(\xi f) \psi
$$

Theorem 6.10 follows, in the same manner as in the proof of Theorem 4.1, from Lemma 3.16. Lemma 6.5 and the next lemma:

Lemma 6.11. For any $f \in D(T)$ the following equations hold:

$$
\begin{aligned}
-T X f+\frac{\lambda}{2}\left\langle\left(Y^{*} J-X^{*}\right) g, f\right\rangle g & =-X \xi f, \\
T J Y f+\frac{\lambda}{2}\left\langle f,\left(Y^{*} J-X^{*}\right) g\right\rangle g & =-J Y \xi f .
\end{aligned}
$$


Remark 6.12. By Lemma 3.16 and the definition of $\xi$, the both sides of (6.5) and (6.6) have meaning.

Proof. Let be $a:=\sqrt{\lambda / D^{\prime}\left(x_{0}\right)}$. Then we can see by the definition of $x_{0}$ and (4.5),

$$
\left(Y^{*} J-X^{*}\right) g=-\Omega_{+} D_{-}\left(T^{2}-E_{0}^{2}\right)^{-1} g+\frac{\beta^{-1 / 2} a}{\lambda} U_{\mathrm{b}}
$$

We have

$$
\begin{aligned}
T T_{ \pm} U_{\mathrm{b}} & =\frac{1}{2}\left(\beta^{1 / 2} T^{1 / 2} U_{\mathrm{b}} \pm \beta^{-1 / 2} T^{3 / 2} U_{\mathrm{b}}\right) \\
& =\frac{1}{2}\left(\beta^{1 / 2} T^{1 / 2} U_{\mathrm{b}} \pm \beta^{3 / 2} T^{-1 / 2} U_{\mathrm{b}} \pm \beta^{-1 / 2} a g\right) .
\end{aligned}
$$

Thus, for any $f \in D(T)$, we have

$$
\begin{aligned}
& -T X f+\frac{\lambda}{2}\left\langle\left(Y^{*} J-X^{*}\right) g, f\right\rangle g \\
= & -T U \Omega_{+}^{*} f-\frac{\lambda}{2}\left\langle D_{-}\left(T^{2}-E_{0}^{2}\right)^{-1} g, \Omega_{+}^{*} f\right\rangle g-T T_{+} P f+\frac{\beta^{-1 / 2} a}{2}\left\langle U_{\mathrm{b}}, f\right\rangle g .
\end{aligned}
$$

Then, by (4.4) and (6.7), we have

$$
\begin{aligned}
-T X f+\frac{\lambda}{2}\left\langle\left(Y^{*} J-X^{*}\right) g, f\right\rangle g & =-U T \Omega_{+}^{*} f-\beta\left\langle U_{\mathrm{b}}, f\right\rangle T_{+} U_{\mathrm{b}} \\
& =-X\left(\Omega_{+} T \Omega_{+}^{*}+\beta P\right) f .
\end{aligned}
$$

Thus we obtain (6.5). Similarly one can prove (6.6).

\subsection{Proof of Theorem 2.10 (2)}

Theorem 6.13. For all $f \in D\left(T^{-1 / 2}\right), \psi \in D\left(\mathrm{~d} \Gamma_{\mathrm{b}}(T)^{1 / 2}\right)$ and $t \in \mathbb{R}$,

$$
\begin{aligned}
e^{i t H(\lambda)} C(f) e^{-i t H(\lambda)} \psi & =C\left(e^{i t \xi} f\right) \psi, \\
e^{i t H(\lambda)} C(f)^{*} e^{-i t H(\lambda)} \psi & =C\left(e^{i t \xi} f\right)^{*} \psi .
\end{aligned}
$$

Proof. These are proved in the same way as in the proof of Theorem 5.1 and Theorem 6.10 .

Lemma 6.14. Let $\Omega:=\mathbb{V}^{-1} \Omega_{0}$ where $\mathbb{V}$ is the unitary operator in Lemma 6.9. Then:

(1) There is an eigenvalue $\tilde{E}_{\mathrm{g}}$ of $H(\lambda)$ and $\Omega$ is an eigenvector of $H(\lambda)$ with eigenvalue $\tilde{E}_{\mathrm{g}}$. 
(2) The following equation holds:

$$
\mathbb{V} H(\lambda) \mathbb{V}^{-1}=\mathrm{d} \Gamma_{\mathrm{b}}(\xi)+\tilde{E}_{\mathrm{g}}
$$

(3) The constant $\tilde{E}_{\mathrm{g}}$ is given as follows:

$$
\tilde{E}_{\mathrm{g}}=E_{\mathrm{g}}-\beta\left\|T_{-} U_{\mathrm{b}}\right\|^{2}
$$

Proof. Parts (1) and (2) can be proved in the same way as in the proof of Theorem 2.10 (1). (3) We have

$$
\tilde{E}_{\mathrm{g}}=\frac{\lambda}{4}\|g\|^{2}-\operatorname{Tr}\left(\xi^{1 / 2} Y^{*} Y \xi^{1 / 2}\right)
$$

in the same way as in the proof of Lemma 5.2. Then, by Lemma 6.5, we have

$$
\xi^{1 / 2} Y^{*} Y \xi^{1 / 2}=\Omega_{+} T^{1 / 2} V^{*} V T^{1 / 2} \Omega_{+}^{*}+\Omega_{+} T^{1 / 2} V^{*} \beta^{1 / 2} T_{-} P+\beta^{1 / 2} P T_{-} V T^{1 / 2} \Omega_{+}^{*}+\beta P T_{-} T_{-} P .
$$

We choose a CONS $\left\{e_{n}\right\}_{n=0}^{\infty} \subset D(T)$ satisfying $e_{0}=U_{\mathrm{b}}$. Then it is easy to see that $\left\{\Omega_{+}^{*} e_{n}\right\}_{n=1}^{\infty}$ is a CONS for $\mathscr{H}$ by Lemma 3.13 . Hence we have

$$
\begin{aligned}
\operatorname{Tr}\left(\xi^{1 / 2} Y^{*} Y \xi^{1 / 2}\right) & =\sum_{n=1}^{\infty}\left\langle e_{n}, \Omega_{+} T^{1 / 2} V^{*} V T^{1 / 2} \Omega_{+}^{*} e_{n}\right\rangle+\beta\left\|T_{-} U_{b}\right\|^{2} \\
& =\operatorname{Tr}\left(T^{1 / 2} V^{*} V T^{1 / 2}\right)+\beta\left\|T_{-} U_{b}\right\|^{2}
\end{aligned}
$$

Thus we obtain (6.8).

In particular, $H(\lambda)$ have eigenvectors as follows:

$$
\phi_{n}:=\mathbb{V}^{-1} A\left(U_{\mathrm{b}}\right)^{* n} \Omega_{0}, H(\lambda) \phi_{n}=\left(n \beta+\tilde{E}_{\mathrm{g}}\right) \phi_{n}, n \in \mathbb{N} \cup\{0\} .
$$

Hence the spectral properties of $H(\lambda)$ as stated in Theorem 2.10 (2) follow.

\section{Analysis in the case $\lambda<\lambda_{\mathrm{c}, 0}$.}

In this section, we show that $H(\lambda)$ is unbounded from above and below.

Theorem 7.1. Let $g \in D\left(T^{-1 / 2}\right)$. Then $H(\lambda)$ is unbounded above for any $\lambda \in \mathbb{R}$. If $\lambda<\lambda_{\mathrm{c}, 0}$, then $H(\lambda)$ is unbounded below too. 
Proof. For any $f \in D(T) \backslash\{0\}$, we set $\psi_{n}:=a_{n} A(f)^{* n} \Omega_{0}, a_{n} \in \mathbb{C} \backslash\{0\}, n \in \mathbb{N} \cup\{0\}$. Then we have the following equations:

$$
\begin{array}{ll}
\mathrm{d} \Gamma_{\mathrm{b}}(T) \psi_{n}=n \frac{a_{n}}{a_{n-1}} A(T f)^{*} \psi_{n-1}, & A(g) \psi_{n}=n\langle g, f\rangle \frac{a_{n}}{a_{n-1}} \psi_{n-1}, \\
\left\|\psi_{n}\right\|^{2}=\left|a_{n}\right|^{2} n !\|f\|^{2 n}, & \left\|A(g)^{*} \psi_{n}\right\|^{2}=\|g\|^{2}\left\|\psi_{n}\right\|^{2}+\left\|A(g) \psi_{n}\right\|^{2} .
\end{array}
$$

Then we have

$$
\left\langle\psi_{n}, H(\lambda) \psi_{n}\right\rangle=\left\|\psi_{n}\right\|^{2}\left(\frac{\lambda}{4}\|g\|^{2}+n \frac{2\left\|T^{1 / 2} f\right\|^{2}+\lambda|\langle g, f\rangle|^{2}}{2\|f\|^{2}}\right) .
$$

We take $f$ such that $\langle g, f\rangle=0$. Then we have $\left\langle\psi_{n}, H(\lambda) \psi_{n}\right\rangle /\left\|\psi_{n}\right\|^{2} \rightarrow \infty$ as $n \rightarrow \infty$ for any $\lambda \in \mathbb{R}$. Thus $H(\lambda)$ is unbounded above for any $\lambda \in \mathbb{R}$.

Let $\phi_{N}:=\sum_{n=0}^{N} \psi_{n}, N=0,1,2, \ldots$ Then we have $\left\|\phi_{N}\right\|^{2}=\sum_{n=0}^{N}\left\|\psi_{n}\right\|^{2}$ and

$$
\begin{aligned}
\left\langle\phi_{N}, H(\lambda) \phi_{N}\right\rangle= & \sum_{n=2}^{N}\left\|\psi_{n}\right\|^{2}\left(\frac{\lambda\|g\|^{2}}{4}+n \frac{2\left\|T^{1 / 2} f\right\|^{2}+\lambda|\langle g, f\rangle|^{2}}{2\|f\|^{2}}+\frac{\lambda}{2} \operatorname{Re} \frac{a_{n-2}{ }^{*}}{a_{n}{ }^{*}} \frac{\langle g, f\rangle^{2}}{\|f\|^{4}}\right) \\
& +\left\|\psi_{1}\right\|^{2}\left(\frac{\lambda\|g\|^{2}}{4}+\frac{\left\|T^{1 / 2} f\right\|^{2}}{\|f\|^{2}}+\frac{\lambda|\langle g, f\rangle|^{2}}{2\|f\|^{2}}\right)+\frac{\lambda\left\|\psi_{0}\right\|^{2}\|g\|^{2}}{4} .
\end{aligned}
$$

Let $a_{0}:=1, a_{n}:=n^{-3 / 4} n !^{-1 / 2}, n \in \mathbb{N}$ and, for any $0<\delta, 0<\varepsilon<1$,

$$
\begin{aligned}
& f=f_{\delta}:=\frac{T^{-1} E((\delta, \infty)) g}{\left\|T^{-1} E((\delta, \infty)) g\right\|}, \\
& c_{\lambda}(\varepsilon, \delta):=\left\|T^{1 / 2} f_{\delta}\right\|^{2}\left\{1+\frac{\lambda}{2}(2-\varepsilon)\left\|T^{-1 / 2} E((\delta, \infty)) g\right\|^{2}\right\} .
\end{aligned}
$$

Then $\sum_{n=0}^{\infty}\left\|\psi_{n}\right\|^{2}$ converges and, for any $N \in \mathbb{N}$,

$$
\left\langle\phi_{N}, H(\lambda) \phi_{N}\right\rangle=\sum_{n=2}^{N}\left\|\psi_{n}\right\|^{2} n c_{\lambda}(\varepsilon, \delta)+\frac{\lambda}{2} \sum_{n=2}^{N}\left\|\psi_{n}\right\|^{2}\left(\frac{a_{n-2}}{a_{n}}-n(1-\varepsilon)\right)\left\langle g, f_{\delta}\right\rangle^{2}+C_{N}
$$

where

$$
C_{N}:=\frac{\lambda\|g\|^{2}}{4} \sum_{n=0}^{N}\left\|\psi_{n}\right\|^{2}+\left\|\psi_{1}\right\|^{2}\left(\left\|T^{1 / 2} f_{\delta}\right\|^{2}+\frac{\lambda}{2}\left\langle g, f_{\delta}\right\rangle^{2}\right) .
$$

For all $0<\delta, 0<\varepsilon<1$, we have

$$
-\frac{2}{\left\|T^{-1 / 2} E((\delta, \infty)) g\right\|^{2}(2-\varepsilon)}<\lambda_{\mathrm{c}, 0} .
$$

The left hand side of 7.2 tends to $\lambda_{c, 0}$ as $\varepsilon, \delta \downarrow 0$. Since $\lambda<\lambda_{c, 0}$, we can take a pair $(\varepsilon, \delta)$ satisfying $c_{\lambda}(\varepsilon, \delta)<0$. We fix such a pair. There is a $n_{0} \in \mathbb{N}$ such that $a_{n-2} / a_{n}-n(1-\varepsilon)>0$ for all $n \geq n_{0}$. Hence we can see that $\left\langle\phi_{N}, H(\lambda) \phi_{N}\right\rangle /\left\|\phi_{N}\right\|^{2}$ tends to $-\infty$ as $N \rightarrow \infty$, because the first term on the right hand side of $(7.1)$ tends to $-\infty$ as $N \rightarrow \infty$. 


\section{Generalization of the $\phi^{2}$-model}

In this section we consider $H(\eta, \lambda)$ defined in Subsection 2.3.

Assumption 8.1. We need the following assumptions:

(1) $f \in D\left(T^{1 / 2}\right)$ and $g \in D\left(T^{-1 / 2}\right) \cap D\left(T^{1 / 2}\right)$,

(2) $f \in D\left(T^{-1}\right)$ and $\operatorname{Re}\left\langle T^{-1} f, g\right\rangle=0$,

(3) $f, g \in D\left(T^{-1}\right)$ and $\operatorname{Re}\left\langle T^{-1} f, g\right\rangle \neq 0$.

We can prove a slight generalization of Theorem 2.10.

Theorem 8.2. Let $\mathscr{H}$ be separable. Then the following (1)-(5) hold:

(1) Suppose that Assumption 2.7 and Assumption 8.1 (2) or (3) hold. Let $\lambda>\lambda_{\mathrm{c}}$. Then there is a unitary operator $\mathbb{U}$ on $\mathscr{F}_{\mathrm{b}}(\mathscr{H})$ such that for all $\eta \in \mathbb{R}$,

$$
\mathbb{U} H(\eta, \lambda) \mathbb{U}^{-1}=\mathrm{d} \Gamma_{\mathrm{b}}(T)+E_{\mathrm{g}}+E_{f, g}
$$

where the constant $E_{f, g} \in \mathbb{R}$ is defined by

$$
E_{f, g}=-\frac{\eta^{2}}{2}\left\|T^{-1 / 2} f\right\|^{2}+\frac{\left(\operatorname{Re}\left\langle T^{-1} f, g\right\rangle\right)^{2} \eta^{2} \lambda}{2\left(1+\lambda\left\|T^{-1 / 2} g\right\|^{2}\right)} .
$$

(2) Suppose that Assumption 2.7 and Assumption 8.1 (2) or (3) hold. Let $E_{0}>0$ and $\lambda>\lambda_{\mathrm{c}}$. Then there are a unitary operator $\mathbb{V}$ on $\mathscr{F}_{\mathrm{b}}(\mathscr{H})$ and a non-negative, injective self-adjoint operator $\xi$ on $\mathscr{H}$ such that, for all $\eta \in \mathbb{R}$,

$$
\mathbb{V} H(\eta, \lambda) \mathbb{V}^{-1}=\mathrm{d} \Gamma_{\mathrm{b}}(\xi)+E_{\mathrm{g}}-E_{\mathrm{b}}+E_{f, g}
$$

(3) Let $T$ be a non-negative, injective self-adjoint operator and suppose that $f$ and $g$ satisfy Assumption 8.1 (1) and (2). Then there is a unitary operator $\mathbb{W}$ on $\mathscr{F}_{\mathrm{b}}(\mathscr{H})$ such that, for all $\eta \in \mathbb{R}$,

$$
\mathbb{W} \overline{H\left(\eta, \lambda_{\mathrm{c}, 0}\right)} \mathbb{W}^{-1}=\overline{H\left(\lambda_{\mathrm{c}, 0}\right)}-\frac{\eta^{2}}{2}\left\|T^{-1 / 2} f\right\|^{2}
$$

(4) Let $T$ be a non-negative, injective self-adjoint operator and suppose that $f$ and $g$ satisfy Assumption 8.1 (1) and (3). Then, for all $\eta \in \mathbb{R} \backslash\{0\}$,

$$
\sigma\left(\overline{H\left(\eta, \lambda_{\mathrm{c}, 0}\right)}\right)=\mathbb{R}, \quad \sigma_{\mathrm{p}}\left(\overline{H\left(\eta, \lambda_{\mathrm{c}, 0}\right)}\right)=\emptyset
$$


(5) Let $T$ be a non-negative, injective self-adjoint operator and suppose that $f$ and $g$ satisfy Assumption 8.1 (1). Moreover, suppose that Assumption 8.1 (2) or (3) holds. Let $\lambda<\lambda_{\mathrm{c}, 0}$. Then, for all $\eta \in \mathbb{R}, \overline{H(\eta, \lambda)}$ is unbounded above and below.

It is easy to see that Theorem 8.2 is proved by the following lemma and facts in Theorem 2.10 .

Lemma 8.3. Let $T$ be a non-negative, injective self-adjont operator, $f \in D\left(T^{-1}\right)$ and $g \in$ $D\left(T^{-1 / 2}\right) \cap D(T)$.

(1) Let $\operatorname{Re}\left\langle T^{-1} f, g\right\rangle=0$. Then there is a unitary operator $\mathbb{U}_{1}$ on $\mathscr{F}_{\mathrm{b}}(\mathscr{H})$ such that for all $\eta, \lambda \in \mathbb{R}$

$$
\mathbb{U}_{1} \overline{H(\eta, \lambda)} \mathbb{U}_{1}^{-1}=\overline{H(\lambda)}-\frac{\eta^{2}}{2}\left\|T^{-1 / 2} f\right\|^{2}
$$

(2) Let $\operatorname{Re}\left\langle T^{-1} f, g\right\rangle \neq 0$ and $g \in D\left(T^{-1}\right)$.

(i) If $\lambda \neq \lambda_{\mathrm{c}, 0}$, then there is a unitary operator $\mathbb{U}_{2}$ on $\mathscr{F}_{\mathrm{b}}(\mathscr{H})$ such that for all $\eta \in \mathbb{R}$,

$$
\mathbb{U}_{2} \overline{H(\eta, \lambda)} \mathbb{U}_{2}^{-1}=\overline{H(\lambda)}+E_{f, g}
$$

(ii) If $\lambda=\lambda_{\mathrm{c}, 0}$, then for all $\eta \in \mathbb{R} \backslash\{0\}$,

$$
\sigma\left(\overline{H\left(\eta, \lambda_{\mathrm{c}, 0}\right)}\right)=\mathbb{R}, \quad \sigma_{\mathrm{p}}\left(\overline{H\left(\eta, \lambda_{\mathrm{c}, 0}\right)}\right)=\emptyset
$$

Proof. Let $\mathbb{U}_{1}:=e^{-i \Phi_{\mathrm{s}}\left(i \eta T^{-1} f\right)}$ for all $\eta \in \mathbb{R}$. Then, by direct calculation, we obtain

$$
\mathbb{U}_{1} H(\eta, \lambda) \mathbb{U}_{1}^{-1}=H(\lambda)-\frac{\eta^{2}}{2}\left\|T^{-1 / 2} f\right\|^{2}-\lambda \eta \kappa \Phi_{\mathrm{s}}(g)+\frac{\lambda}{2} \eta^{2} \kappa^{2}
$$

on $\mathscr{F}_{\mathrm{b}, \text { fin }}(D(T))$ for all $\eta, \lambda \in \mathbb{R}$, where $\kappa:=\operatorname{Re}\left\langle T^{-1} f, g\right\rangle$. In the case of (1), we have (8.1) by $\kappa=0$ and a limit argument. Next, we prove (2). We assume that $g \in D\left(T^{-1}\right)$ and $\operatorname{Re}\left\langle T^{-1} f, g\right\rangle \neq 0$. Let $\mathbb{V}_{1}:=e^{i \Phi_{\mathrm{s}}\left(i \alpha T^{-1} g\right)}$ for any $\alpha \in \mathbb{R}$ and define a unitary operator $\mathbb{U}_{2}:=\mathbb{V}_{1} \mathbb{U}_{1}$. Then

$$
\begin{aligned}
\mathbb{U}_{2} H(\eta, \lambda) \mathbb{U}_{2}^{-1}= & H(\lambda)+\left(\alpha+\lambda \alpha\left\|T^{-1 / 2} g\right\|^{2}-\lambda \eta \kappa\right) \Phi_{\mathrm{s}}(g) \\
& -\frac{\eta^{2}}{2}\left\|T^{-1 / 2} f\right\|^{2}+\frac{\lambda}{2} \eta^{2} \kappa^{2}+\frac{\alpha}{2}\left\|T^{-1 / 2} g\right\|^{2}\left(\alpha+\lambda \alpha\left\|T^{-1 / 2} g\right\|^{2}-2 \lambda \eta \kappa\right)
\end{aligned}
$$

on $\mathscr{F}_{\mathrm{b}, \text { fin }}(D(T))$ in the same way as in 8.3$)$. For $\lambda \neq \lambda_{\mathrm{c}, 0}$, let $\alpha=\lambda \eta \kappa\left(1+\lambda\left\|T^{-1 / 2} g\right\|^{2}\right)^{-1}$. Then we obtain

$$
\mathbb{U}_{2} \overline{H(\eta, \lambda)} \mathbb{U}_{2}^{-1}=\overline{H(\lambda)}-\frac{\eta^{2}}{2}\left\|T^{-1 / 2} f\right\|^{2}+\frac{\lambda \eta^{2} \kappa^{2}}{2\left(1+\lambda\left\|T^{-1 / 2} g\right\|^{2}\right)}
$$


by a limit argument. If $\lambda=\lambda_{\mathrm{c}, 0}$, then, for all $\eta, \alpha \in \mathbb{R}$, we have

$$
\mathbb{U}_{2} \overline{H\left(\eta, \lambda_{\mathrm{c}, 0}\right)} \mathbb{U}_{2}^{-1}=\overline{H_{g}\left(-\kappa \eta \lambda_{\mathrm{c}, 0}, \lambda\right)}-\frac{\eta^{2}}{2}\left\|T^{-1 / 2} f\right\|^{2}+\frac{\lambda_{\mathrm{c}, 0} \eta^{2} \kappa^{2}}{2}+\kappa \eta \alpha
$$

in the same way as in 8.4), where $H_{g}\left(\nu, \lambda_{\mathrm{c}, 0}\right):=H\left(\lambda_{\mathrm{c}, 0}\right)+\nu \Phi_{\mathrm{s}}(g)$ for all $\nu \in \mathbb{R}$. It is easy to see that $\sigma\left(\overline{H_{g}\left(\nu, \lambda_{\mathrm{c}, 0}\right)}\right)=\mathbb{R}$ and $\sigma_{\mathrm{p}}\left(\overline{H_{g}\left(\nu, \lambda_{\mathrm{c}, 0}\right)}\right)=\emptyset$ for all $\nu \in \mathbb{R} \backslash\{0\}$, because $\mathbb{V}_{1} \overline{H_{g}\left(\nu, \lambda_{\mathrm{c}, 0}\right)} \mathbb{V}_{1}^{-1}=$ $\overline{H_{g}\left(\nu, \lambda_{c, 0}\right)}+\nu \alpha\left\|T^{-1 / 2} g\right\|^{2}$ and $\alpha \in \mathbb{R}$ is arbitrary. Hence we have 8.2 .

Remark 8.4. If $\mathscr{H}$ is separable, then the condition $g \in D\left(T^{-1 / 2}\right) \cap D(T)$ in the above lemma is weakened to the condition $g \in D\left(T^{-1 / 2}\right) \cap D\left(T^{1 / 2}\right)$.

\section{Appendix}

In this section, we recall some known facts in Fock space theory. Let $T$ be a non-negative, injective self-adjoint operator on $\mathscr{H}$.

Lemma 9.1. [5, Theorem 5.16.]

Let $f \in D\left(T^{-1 / 2}\right)$ and $\psi \in D\left(\mathrm{~d} \Gamma_{\mathrm{b}}(T)^{1 / 2}\right)$. Then $\psi \in D(A(f)) \cap D\left(A(f)^{*}\right)$ and the following inequalities hold:

$$
\begin{aligned}
\|A(f) \psi\| & \leq\left\|T^{-1 / 2} f\right\|\left\|\mathrm{d} \Gamma_{\mathrm{b}}(T)^{1 / 2} \psi\right\|, \\
\left\|A(f)^{*} \psi\right\|^{2} & \leq\left\|T^{-1 / 2} f\right\|^{2}\left\|\mathrm{~d} \Gamma_{\mathrm{b}}(T)^{1 / 2} \psi\right\|^{2}+\|f\|^{2}\|\psi\|^{2} .
\end{aligned}
$$

Lemma 9.2. [5, Proposition 5.10.] For any $f \in D(T)$, the following commutation relations on $\mathscr{F}_{\mathrm{b}, \mathrm{fin}}(D(T))$ :

$$
\left[\mathrm{d} \Gamma_{\mathrm{b}}(T), A(f)\right]=-A(T f), \quad\left[\mathrm{d} \Gamma_{\mathrm{b}}(T), A(f)^{*}\right]=A(T f)^{*}
$$

Lemma 9.3. [5, Lemma 5.21.] For any $t \in \mathbb{R}, f \in \mathscr{H}$, the following equation holds:

$$
e^{i t \mathrm{~d} \Gamma_{\mathrm{b}}(T)} A(f)^{\sharp} e^{-i t \mathrm{~d} \Gamma_{\mathrm{b}}(T)}=A\left(e^{i t T} f\right)^{\sharp} .
$$

Lemma 9.4. [5, Theorem 5.21.] Assume that $\mathscr{H}$ be separable. Let $T$ be a non-negative, injective self-adjoint operator and $\left\{e_{n}\right\}_{n=1}^{\infty} \subset D\left(T^{1 / 2}\right)$ be a CONS of $\mathscr{H}$. Then, for any $\psi \in D\left(\mathrm{~d} \Gamma_{\mathrm{b}}(T)^{1 / 2}\right), \sum_{n=1}^{\infty}\left\|A\left(T^{1 / 2} e_{n}\right) \psi\right\|^{2}$ converges and following equation holds:

$$
\sum_{n=1}^{\infty}\left\|A\left(T^{1 / 2} e_{n}\right) \psi\right\|^{2}=\left\|\mathrm{d} \Gamma_{\mathrm{b}}(T)^{1 / 2} \psi\right\|^{2}
$$




\section{References}

[1] Arai A., On a model of a harmonic oscillator coupled to a quantized, massless, scalar field. I, J. Math. Phys. 22 (1981), 2539-2548.

[2] Arai A., Spectral analysis of a quantum harmonic oscillator coupled to infinitely many scalar bosons, J. Math. Anal. Appl. 140 (1989), 270-288.

[3] Arai A., Fock Spaces and Quantum Fields, Nippon Hyoronsya, 2000, in Japanese.

[4] Arai A., A note on mathematical analysis of a pair-interaction model in quantum field theory, Unpublished, 2017.

[5] Arai A., Analysis on Fock Spaces and Mathematical Theory of Quantum Fields, World Scientific, Singapore, 2018.

[6] Berezin F. A., The Method of Second Quantization, Academic Press, New York, 1966.

[7] Dereziński J., Bosonic quadratic Hamiltonians, J. Math. Phys. 58 (2017), 121101-45.

[8] Henley E. M. and Thirring W., Elementary Quantum Field Theory, McGraw-Hill Book Company, New York, 1962.

[9] Hiroshima F., Sasaki I, Spohn H. and Suzuki A., Enhanced Binding in Quantum Field Theory, COE Lecture Note Vol.38, IMI, Kyushu University, 2012.

[10] Miyao T. and Sasaki I., Stability of discrete ground state, Hokkaido Math. J. 34 (2005), 689-717.

[11] Nam P. T., Napiórkowski M. and Solovej J. P., Diagonalization of bosonic quadractic Hamiltonians by Bogoliubov transformations, J. Func. Anal, 270 (2016), 4340-4368.

[12] Reed M. and Simon B., Methods of Modern Mathematical Physics I: Functional Analysis, Academic Press, New York, 1979.

[13] Ruijsenaars S. N. M., On Bogoliubov transformations. II. The general case, Ann. Phys. 116 (1978), 105-134.

[14] Shale D., Linear symmetries of free boson fields, Trans. Amer. Math. Soc. 103 (1962), 149-167.

[15] Teranishi N., Self-adjointness of the generalized spin-boson Hamiltonian with a quadratic boson interaction, Hokkaido Math. J. 44, no. 3 (2015), 409-423. 
[16] Titchmarsh E. C., Introduction to the Theory of Fourier Integrals, Oxford U.P., Amen House, London, 1937. 